\title{
Shot- and angle-domain wave-equation traveltime inversion of reflection data: Synthetic and field data examples
}

\author{
Sanzong Zhang ${ }^{1}$, Yi Luo ${ }^{2}$, and Gerard Schuster ${ }^{1}$
}

\begin{abstract}
Full-waveform inversion requires the accurate simulation of the dynamics and kinematics of wave propagation. This is difficult in practice because the amplitudes cannot be precisely reproduced for seismic waves in the earth. Waveequation reflection traveltime tomography (WT) is proposed to avoid this problem by directly inverting the reflectiontraveltime residuals without the use of the high-frequency approximation. We inverted synthetic traces and recorded seismic data for the velocity model by WT. Our results demonstrated that the wave-equation solution overcame the high-frequency approximation of ray-based tomography, was largely insensitive to the accurate modeling of amplitudes, and mitigated problems with ambiguous event identification. The synthetic examples illustrated the effectiveness of the WT method in providing a highly resolved estimate of the velocity model. A real data example from the Gulf of Mexico demonstrated these benefits of WT, but also found the limitations in traveltime residual estimation for complex models.
\end{abstract}

\section{INTRODUCTION}

Full-waveform inversion (FWI) is a high-resolution model-building method that finds the optimal velocity model by minimizing a data-domain misfit function. The problem with FWI is that its objective function can be highly nonlinear with respect to velocity perturbations. This nonlinearity is largely characterized by cycle skipping between the predicted and recorded data, which results from a poor starting velocity model. To mitigate these difficulties, image-domain methods, such as wave-equation migration velocity analysis (WEMVA), have been proposed to focus or flatten the different types of common-image gathers (CIGs) (Symes and Kern,
1994; Sava and Biondi, 2004; Shen and Symes, 2008; Yang and Sava, 2011, 2013). A benefit of WEMVA is that waveform fitting, including amplitude and phase, is largely avoided.

Migration velocity analysis (MVA) is another type of velocity inversion, which estimates the interval velocity model by residual moveout analysis. Al-Yahya (1987) helps to introduce migrationbased velocity analysis, which assumes that the correct velocity model should horizontally align the migration images in commonreceiver gathers regardless of structure. He introduces an iterative inversion method, which uses an initial velocity model for migration, and the errors in the velocity model are estimated from the curvature of events in the CIGs. A semblance scanning method is used to search for the curvature of the misaligned migration events.

In addition to ray-based MVA, there are many variations of WEMVA, including the inversion of time lags (Yang and Sava, 2011, 2013) and space lags (Shen and Symes, 2008; Zhang and Schuster, 2012) in CIGs for estimating the velocity model. However, for Yang and Sava (2011), the conversion from the time lag to an image perturbation may not be valid with large time lags, and a complex velocity model can spoil accurate picking of the time lag in the time-shift extended image (Wang et al., 2009). To partly remedy this problem, Almomin (2011) minimizes the depth lags, which maximize the crosscorrelation of the observed image with a reference image in the reflection angle gathers. Because the true image depth is difficult to determine in practice, it can be unduly biased by the reference image. The merit of this method is that it is less sensitive to the amplitude of the observed data and is mostly immune to cycle-skipping problems. Similarly, Yang and Sava (2013) apply the same idea to the time-lag images, and the model is updated by focusing the image energy at zero time lag. In practice, it is sometimes difficult to estimate the time lag from the time-lag extended image due to image defocusing, especially in complex salt models. In addition to the time-lag method, the subsurface offset or space lag can also be inverted for the velocity model (Shen and Symes, 2008; Zhang and Schuster, 2012). The objective function of these methods

\footnotetext{
Manuscript received by the Editor 12 May 2014; revised manuscript received 29 December 2014; published online 26 May 2015.

${ }^{1}$ King Abdullah University of Science and Technology, Center for Subsurface Imaging and Fluid Modeling, Thuwal, Saudi Arabia. E-mail: sanzong.zhang@ kaust.edu.sa; gerard.schuster@kaust.edu.sa.

EXPEC ARC, Saudi Aramco, Dhahran, Saudi Arabia. E-mail: yi.luo@aramco.com.

(C) 2015 Society of Exploration Geophysicists. All rights reserved.
} 
does not minimize the image residual like differential semblance analysis, instead it minimizes the space lag directly. The benefit is that the space-lag misfit function enjoys a more quasilinear relationship with respect to velocity perturbations (Zhang and Schuster, 2012).

As an alternative to the image-domain methods, Zhang et al. $(2011,2012,2015)$ present the wave-equation reflection traveltime tomography (WT) method for inverting reflection traveltimes. This method exhibits some advantages compared with ray-based tomography and FWI. Compared with ray-based tomography, the WT method does not require a high-frequency approximation, exhibits moderate resolution with complex structures, and the conversion from the depth residual to the time residual is not required. The WT gradient function is approximately equivalent to that of FWI if the starting velocity model is close to the true velocity model (Luo and Schuster, 1991). Although the starting velocity model is far from the true velocity model, FWI has a cycle-skipping problem because the observed arrival can be delayed from the predicted arrival by more than a cycle. The WT method can mitigate cycleskipping problems and update the velocity by smearing the redatumed data delayed and weighted by the reflection-traveltime residual. Another benefit of WT over FWI is that amplitude fitting is avoided. A robust estimate of the reflection-traveltime residual is key for the successful use of the WT method.

We now present the numerical results for applying WT (Zhang et al., 2015) to synthetic traces and to marine data recorded in the Gulf of Mexico. The synthetic results show how WT has better convergence properties than FWI when the starting model is poor. They also illustrate that the angle-domain crosscorrelation function provides an efficient way to estimate the traveltime residual for complex models. Finally, marine data recorded in the Gulf of

a)
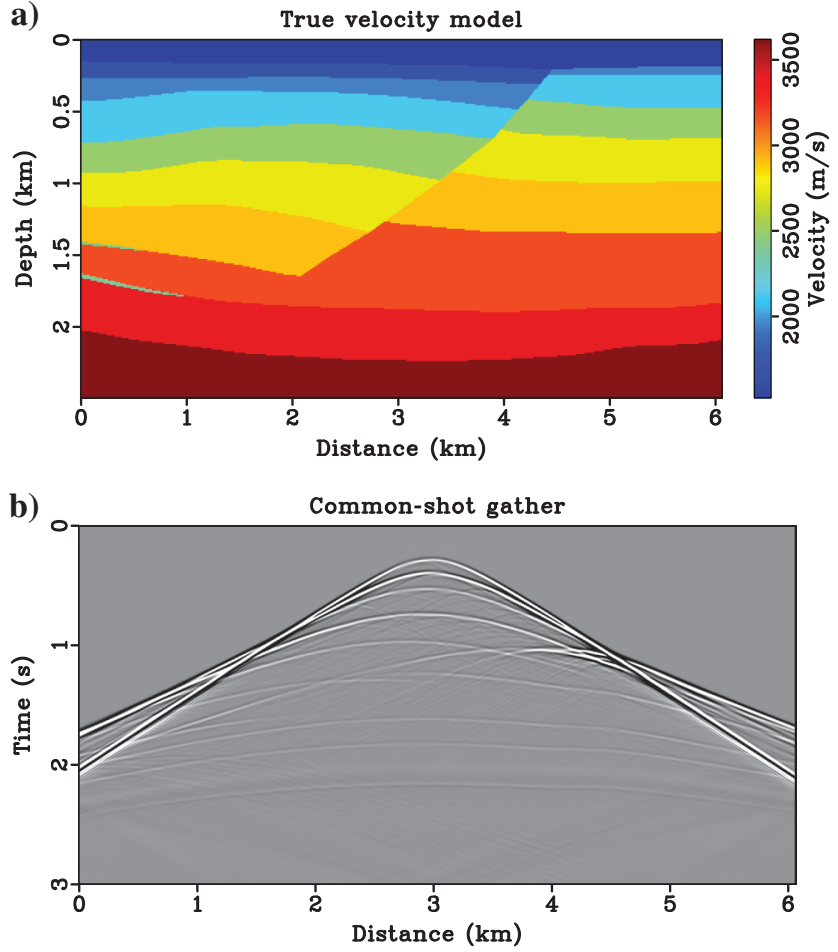

Figure 1. (a) A simple fault model and (b) a common-shot gather.
Mexico are inverted by the angle-domain WT method, and the WT tomograms are compared with the FWI results.

\section{METHOD}

The theory of the WT method is presented in Zhang et al. (2015), where reflection traveltimes are inverted by back projecting traveltime residuals along the associated wavepaths. Wavepaths are computed by numerical solutions to the wave equation, and this avoids the high-frequency approximation of ray-tracing tomography. The workflow and formulas (Zhang et al., 2015) for implementing WT are described as follows:

1) Calculate the shot-domain or angle-domain crosscorrelation function. This step requires the current starting velocity model and the observed data. The migration method can be either reverse time migration (RTM) or the one-way wave-equation
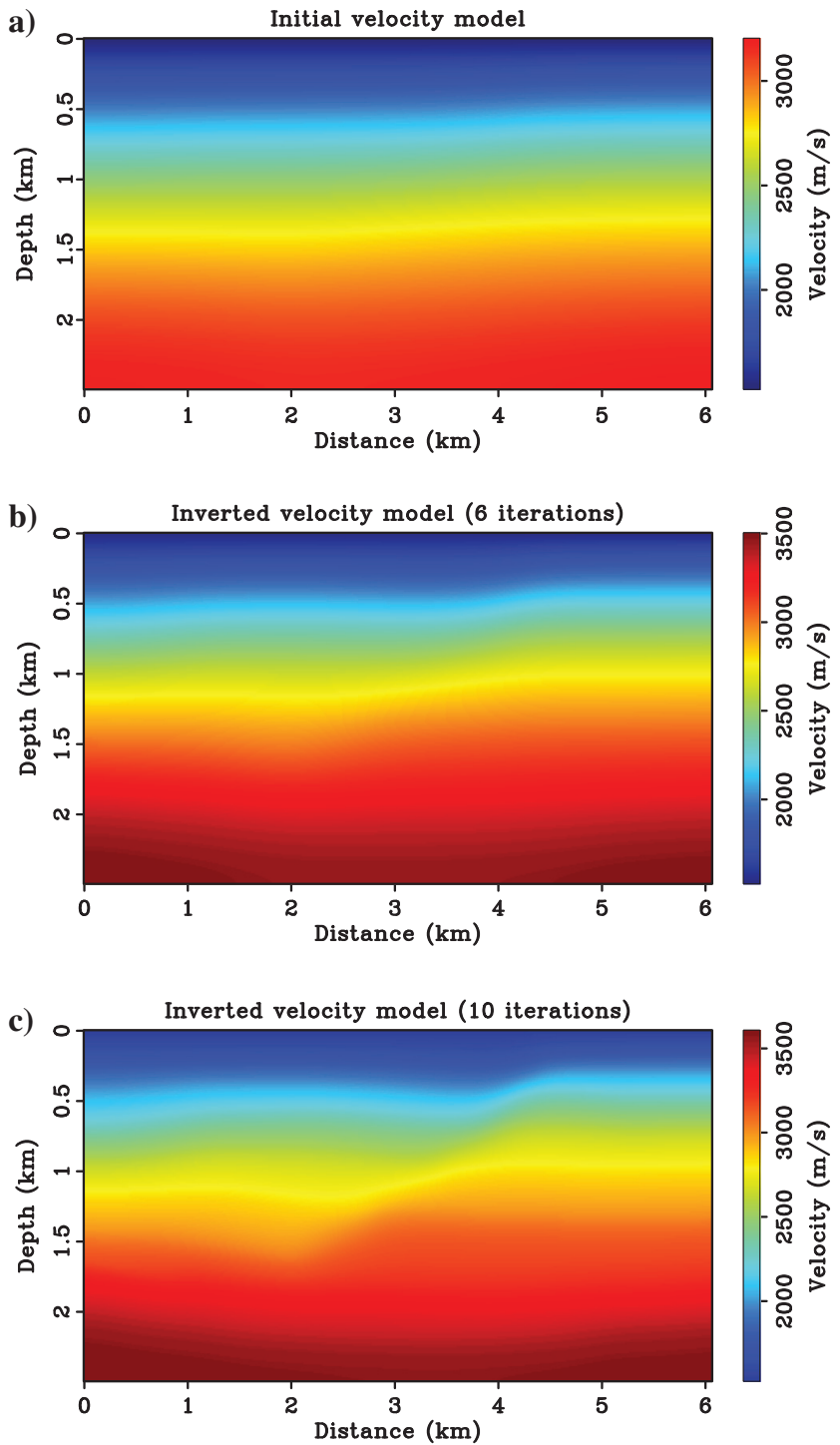

Figure 2. (a) The initial velocity model and (b and c) the inverted tomograms after 6 and $10 \mathrm{WT}$ iterations, respectively. 
migration. The shot-domain crosscorrelation between the forward-propagated wavefield $p_{s}\left(\mathbf{x}, t \mid \mathbf{x}_{s}\right)$ and backward-propagated wavefield $p_{g}\left(\mathbf{x}, t \mid \mathbf{x}_{s}\right)$ at a trial image point $\mathbf{x}$ in the subsurface is given by

$$
f\left(\mathbf{x}, \mathbf{x}_{s}, \tau\right)=\int p_{s}\left(\mathbf{x}, t+\tau \mid \mathbf{x}_{s}\right) p_{g}\left(\mathbf{x}, t \mid \mathbf{x}_{s}\right) d t
$$

where $\tau\left(\mathbf{x}, \mathbf{x}_{s}\right)$ is the time lag of the crosscorrelation function $f\left(\mathbf{x}, \mathbf{x}_{s}, \tau\right)$. The time integration is over the entire trace. The angle-domain crosscorrelation function is a)

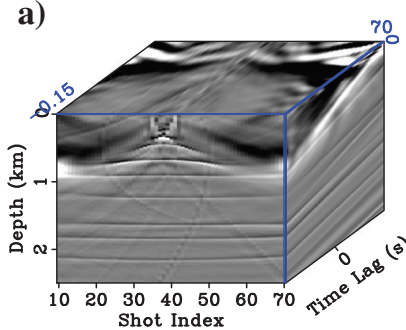

c)

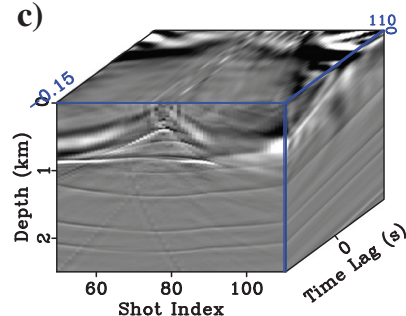

b)

d)

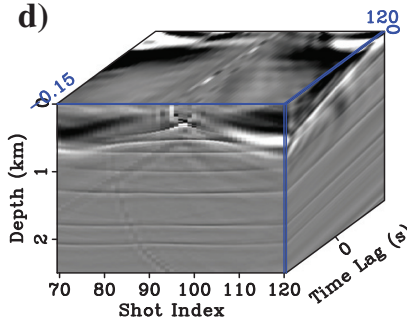

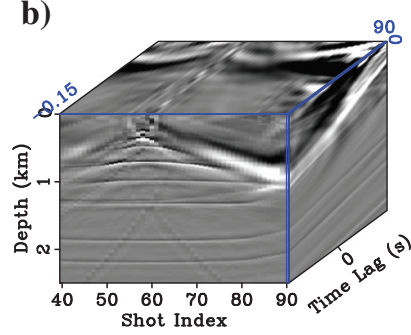

Figure 3. (a-d) The shot-domain crosscorrelation functions generated from the initial velocity model. The locations are indicated by the four black lines in Figure 1a.
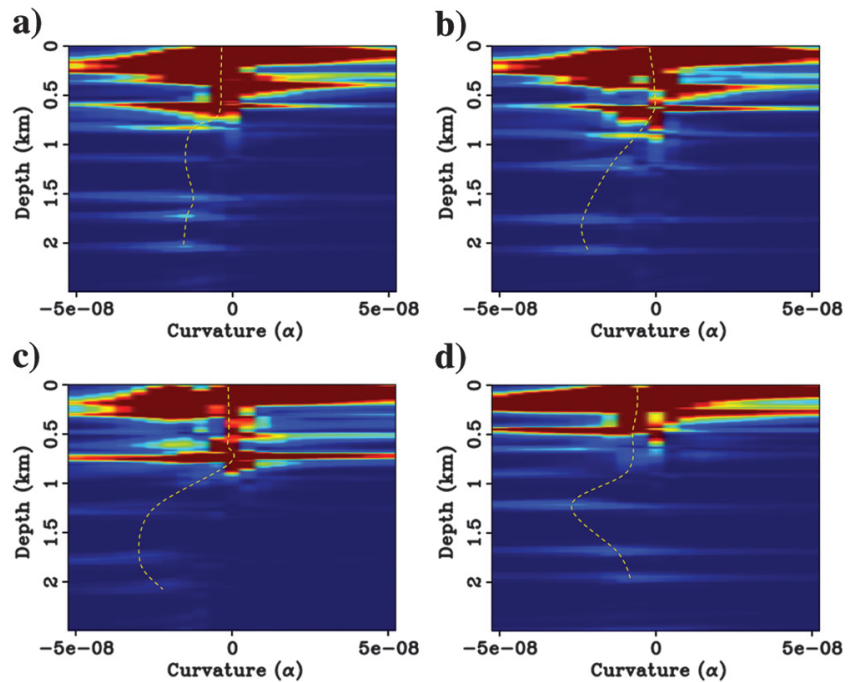

d)

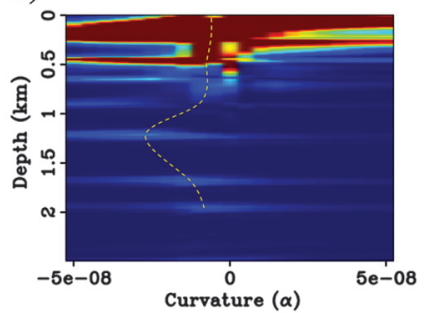

Figure 4. Semblance spectra calculated from Figure 3 for the WT first iteration at offsets of $x=$ (a) 1.8 , (b) 2.8 , (c) 3.8 , and (d) $4.8 \mathrm{~km}$. The yellow dashed lines indicate the maximum energy cluster for the curvature as a function of reflection depth.

$$
\begin{aligned}
f(\mathbf{x}, \theta, \tau)= & \int d \mathbf{x}_{s} \int d h \int p_{s}(x-h, z+h \tan \theta, t \\
& \left.+\tau \mid \mathbf{x}_{s}\right) p_{g}\left(x+h, z+h \tan \theta, t \mid \mathbf{x}_{s}\right) d t,
\end{aligned}
$$

where $h$ is the subsurface offset and $\theta$ is the reflection angle. The benefit in correlating the source trace with the redatumed trace at depth $\mathbf{x}$ is that the reflections are more focused with a higher signal-to-noise ratio at depth than they are at the original recording plane. The forward-modeled wavefield $p_{s}\left(\mathbf{x}, t \mid x_{s}\right)$ is initiated by the line source at $\mathbf{x}_{s}$

$$
p_{s}\left(\mathbf{x}, t \mid \mathbf{x}_{s}\right)=w(t) * g\left(\mathbf{x}, t \mid \mathbf{x}_{s}, 0\right)
$$

a)

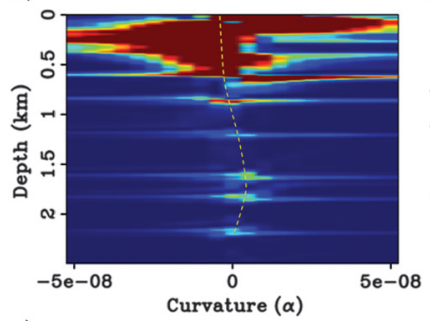

c)

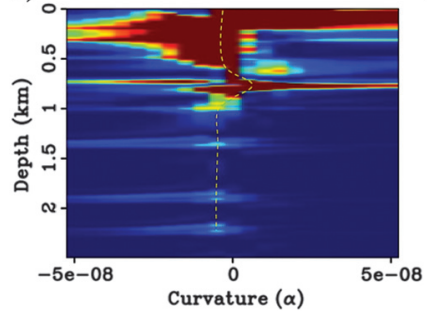

b)

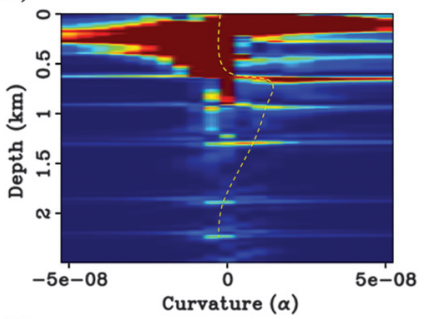

d)

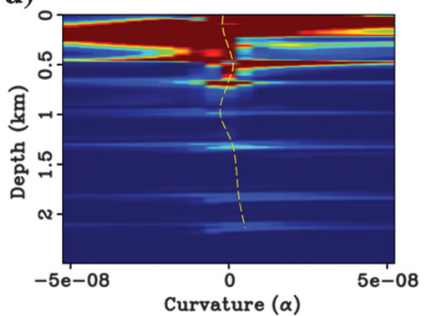

Figure 5. The same as the previous figure except after six iterations. a)

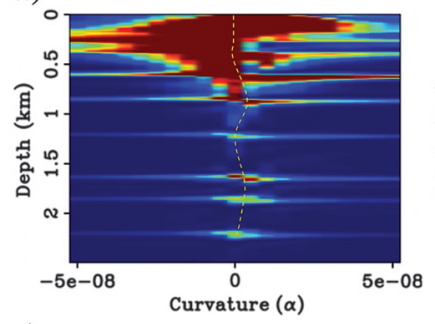

c)

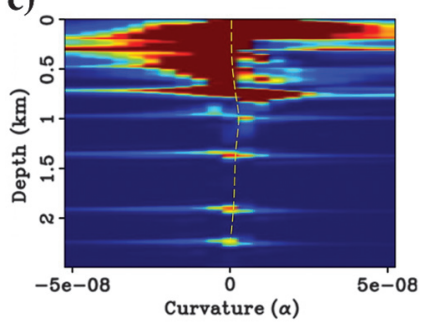

b)

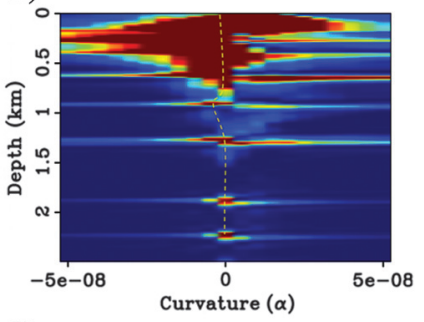

d)

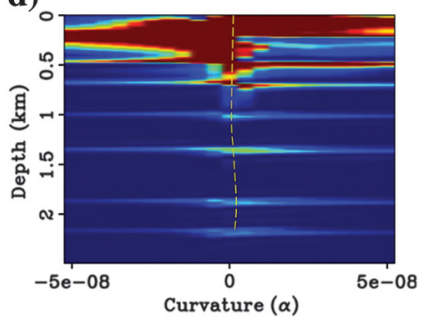

Figure 6. The same as the previous figure except after 10 iterations. 
where $w(t)$ is the source wavelet; the symbol $*$ denotes convolution; and $p_{g}\left(\mathbf{x}, t \mid \mathbf{x}_{s}\right)$ is the backward-propagated wavefield computed by time-reversed propagation of the observed data $p\left(\mathbf{x}_{g}, t \mid \mathbf{x}_{s}\right)_{\text {obs }}$

$$
p_{g}\left(\mathbf{x}, t \mid \mathbf{x}_{s}\right)=\int p\left(\mathbf{x}_{g}, t \mid \mathbf{x}_{s}\right)_{\mathrm{obs}} * g\left(\mathbf{x},-t \mid \mathbf{x}_{g}, 0\right) d \mathbf{x}_{g}
$$

We also denote $p_{s}\left(\mathbf{x}, t \mid \mathbf{x}_{s}\right)$ as the redatumed source and $p_{g}\left(\mathbf{x}, t \mid \mathbf{x}_{s}\right)$ as the redatumed data.

2) Calculate the semblance spectrum. The equation used to fit the shot-domain traveltime residual curve is given by

$$
\Delta \tau=\alpha(\Delta x)^{2}
$$
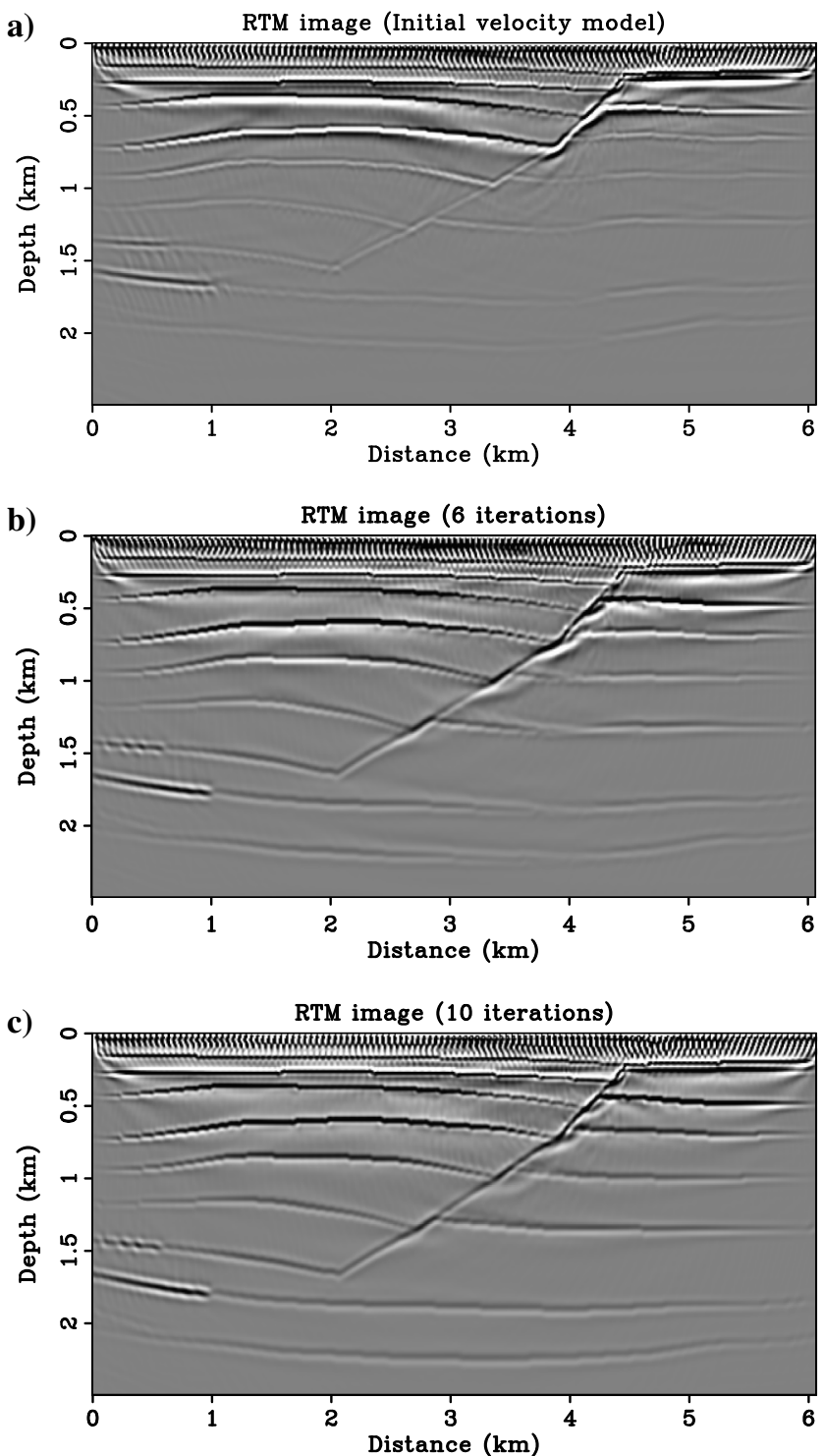

Figure 7. (a-c) The RTM images using the initial velocity and inverted velocity models in Figure 2.
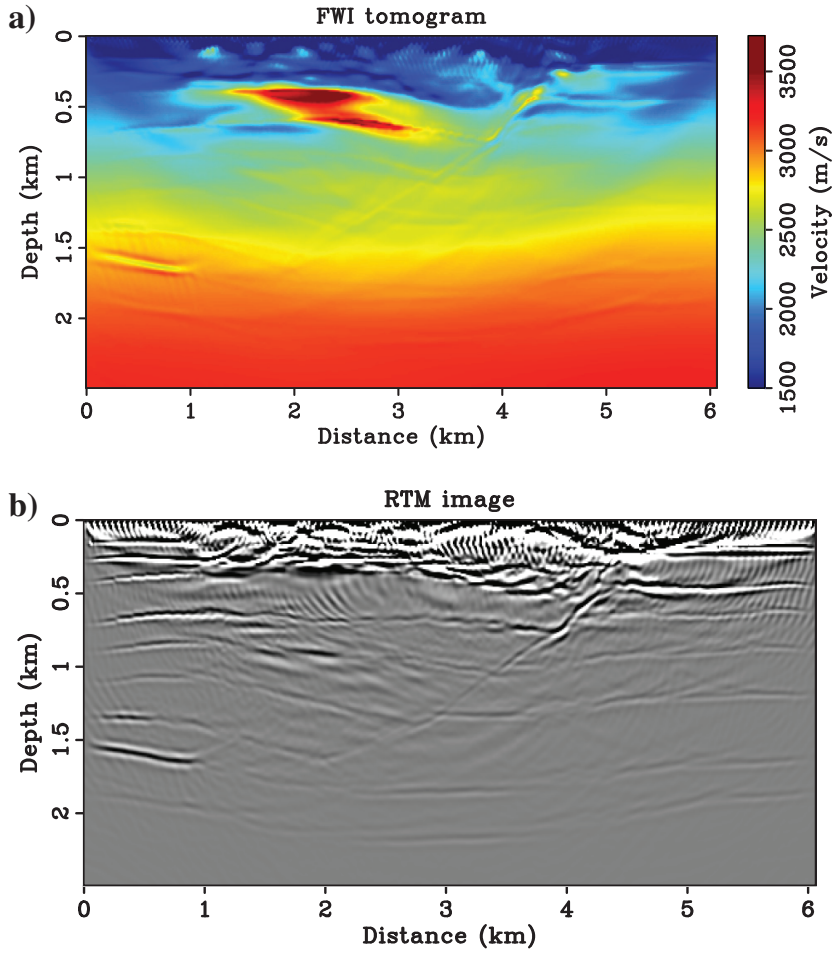

Figure 8. ( $\mathrm{a}$ and $\mathrm{b}$ ) The FWI tomograms and the associated RTM images.
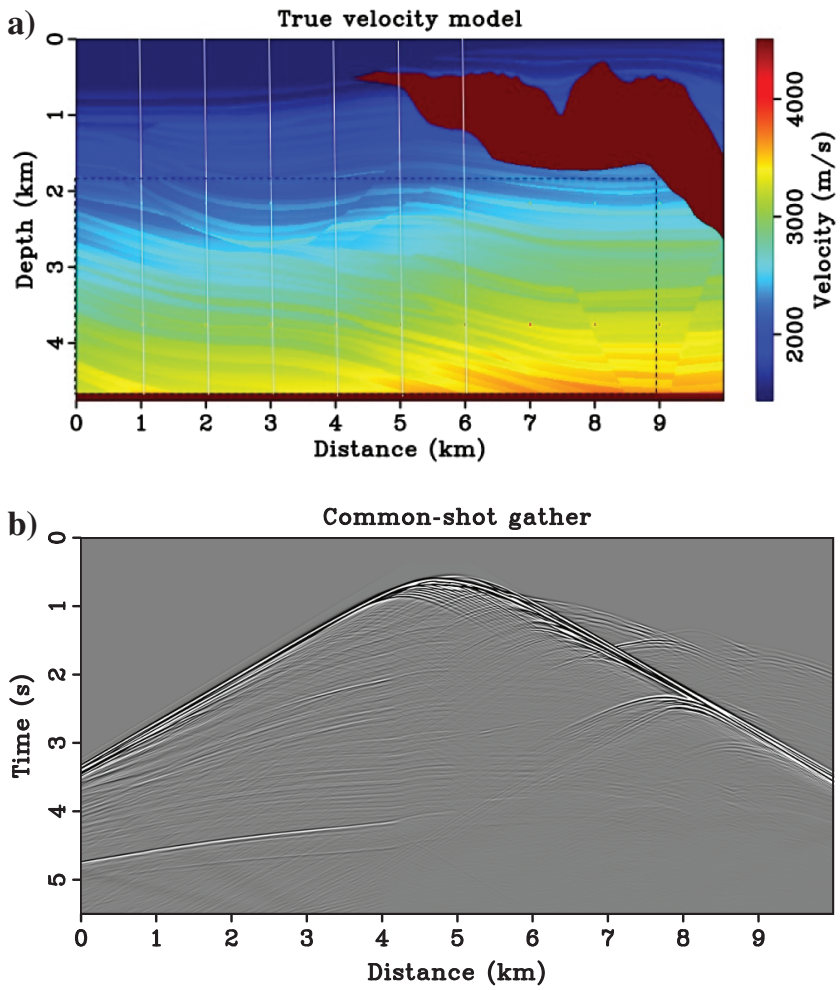

Figure 9. (a) Sigsbee model and (b) common-shot gather. 
where $\alpha$ is the curvature of the hyperbolic curve, $\Delta \tau$ is the time lag that maximizes the amplitude of the crosscorrelation function, and $\Delta x$ is the horizontal distance between the location of the reflection point and the source. The equation used to fit the angle-domain reflection-traveltime residual curve is given by

$$
\Delta \tau=\alpha(\tan \theta)^{2}
$$

where $\alpha$ is the curvature parameter and $\theta$ is the reflection angle.

3) Pick the maximum energy in the semblance spectrum and calculate the reflection-traveltime residual.

4) Calculate the WT gradient function in the shot domain, which is given by

$$
\gamma\left(\mathbf{x}^{\prime}\right)=\gamma_{1}\left(\mathbf{x}^{\prime}\right)+\gamma_{2}\left(\mathbf{x}^{\prime}\right),(7)
$$
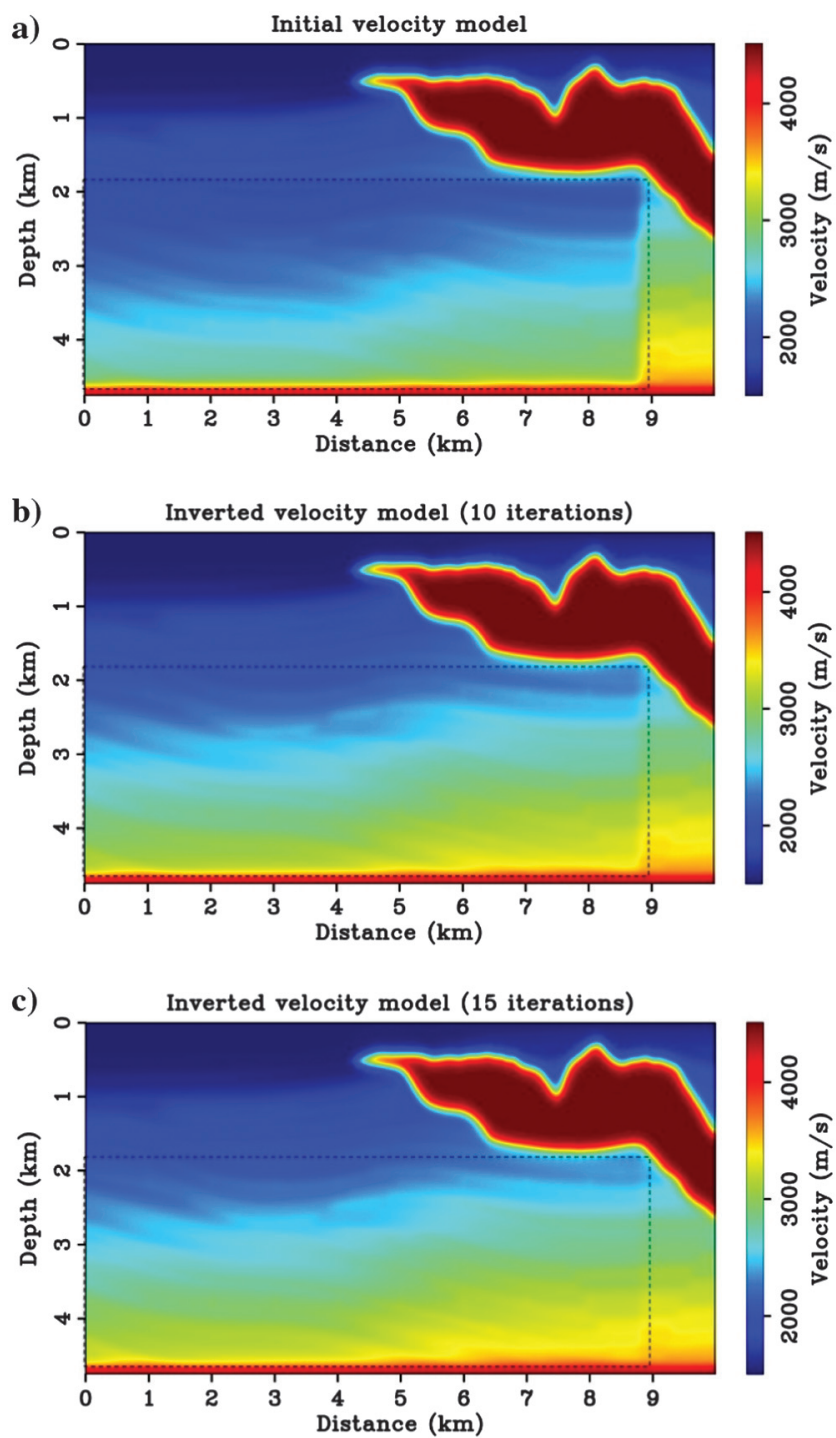

Figure 10. (a) The initial velocity model and (b and c) the inverted tomograms after 10 and 15 iterations. where

$$
\begin{aligned}
& \gamma_{1}\left(\mathbf{x}^{\prime}\right)= \frac{2}{c\left(\mathbf{x}^{\prime}\right)^{3}} \\
& \times \sum_{\mathbf{x}_{s}} \sum_{\mathbf{x}} \int \overbrace{\left[\int \frac{\Delta \tau}{E} \dot{p}_{g}\left(\mathbf{x}_{g}, t \mid \mathbf{x}_{s}\right) * g\left(\mathbf{x}^{\prime},-t \mid \mathbf{x}_{g}, 0\right) d \mathbf{x}_{g}\right]}^{\text {back propagation of the observed data }} \\
& \\
& \overbrace{\left[\ddot{p}_{s}\left(\mathbf{x}, t+\Delta \tau \mid \mathbf{x}_{s}\right) * g\left(\mathbf{x}^{\prime}, t \mid \mathbf{x}, 0\right)\right]}^{\text {forward propagation of the redatumed source }} d t
\end{aligned}
$$

and
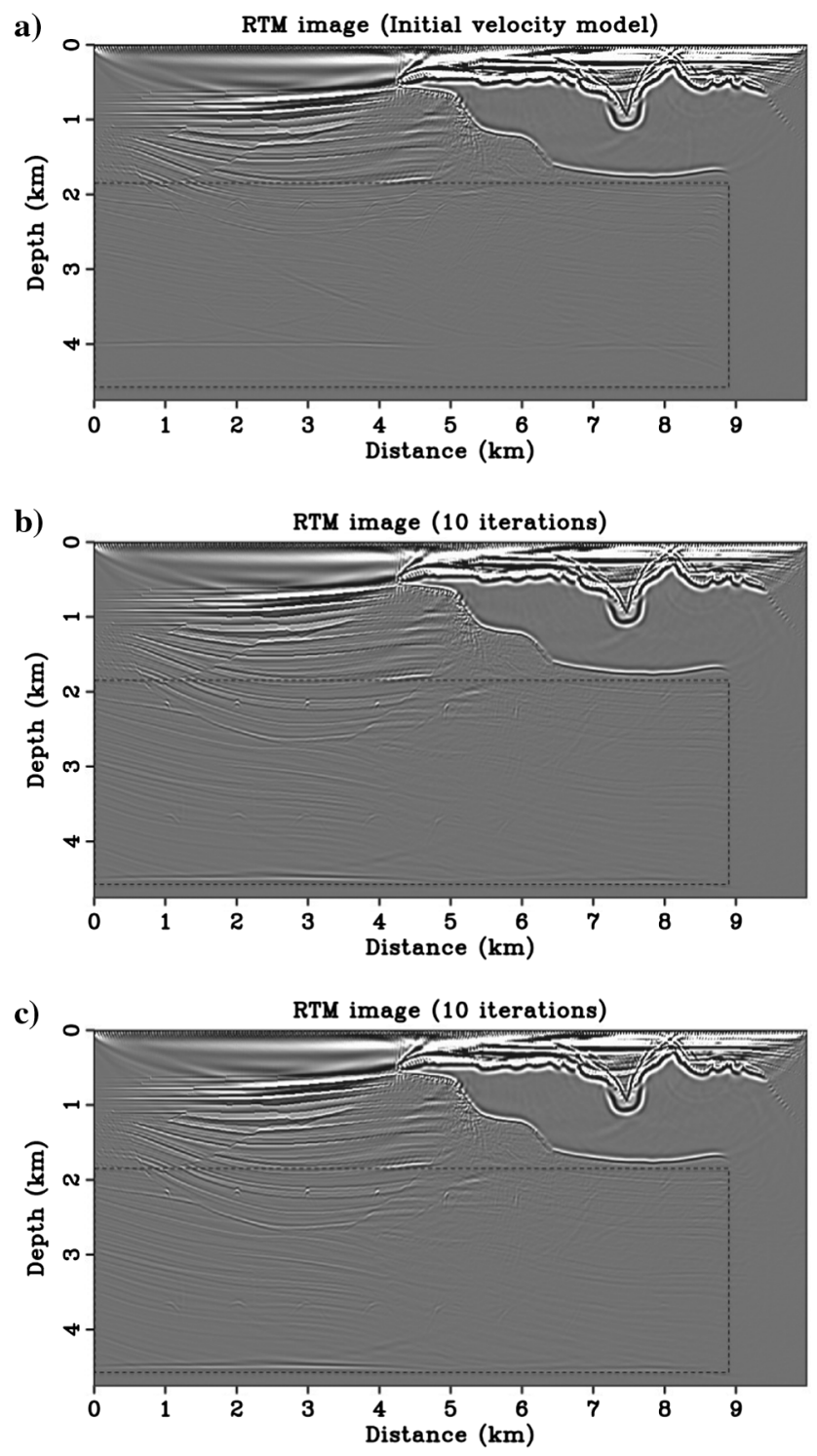

Figure 11. An RTM images using the velocity models in Figure 10. 

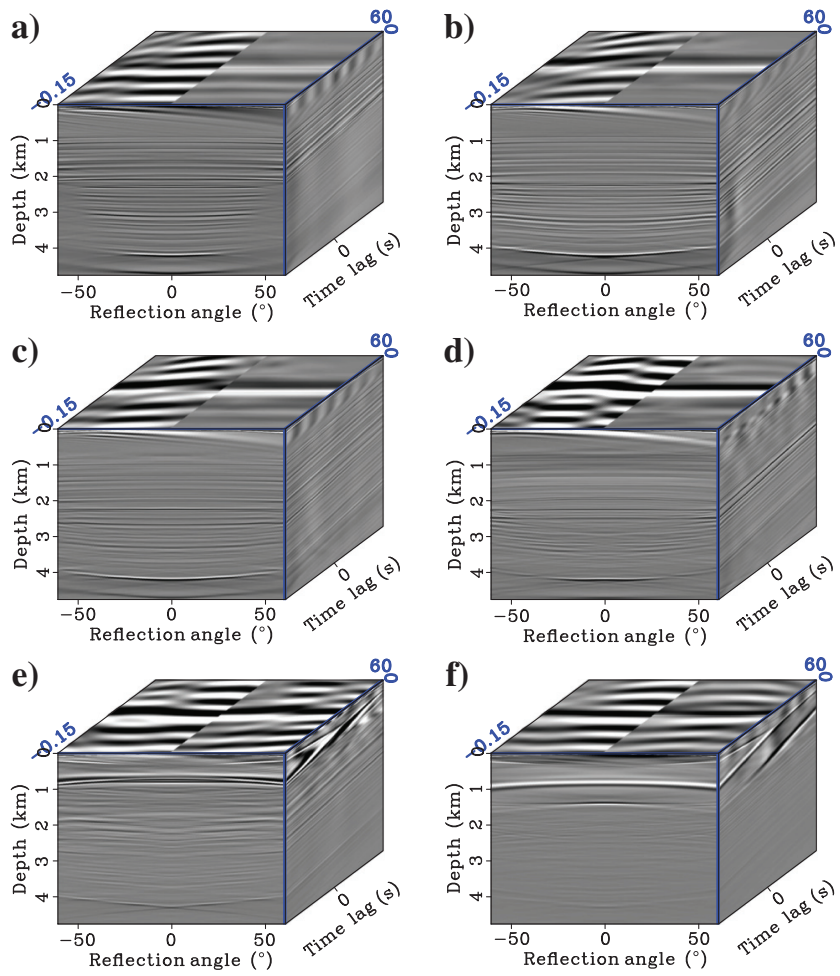

Figure 12. Angle-domain crosscorrelation functions generated from the initial velocity model. The offsets are indicated by the six lines from left to right in Figure 9a.

a)

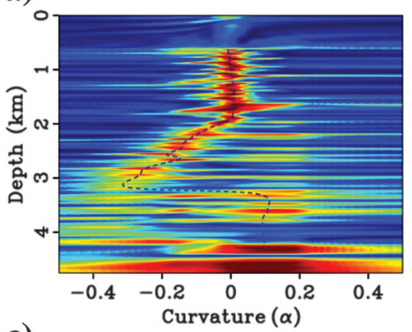

c)

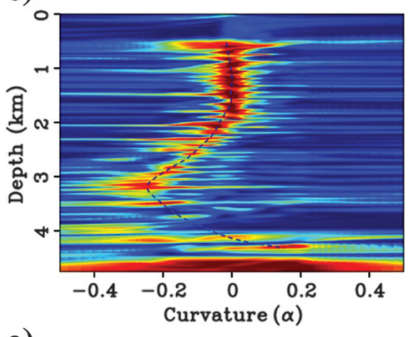

e)

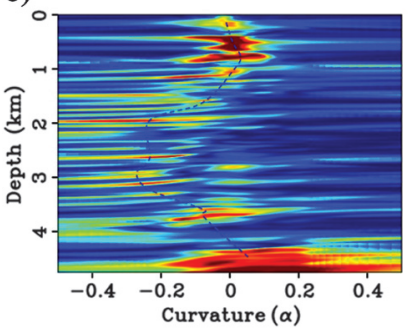

b)

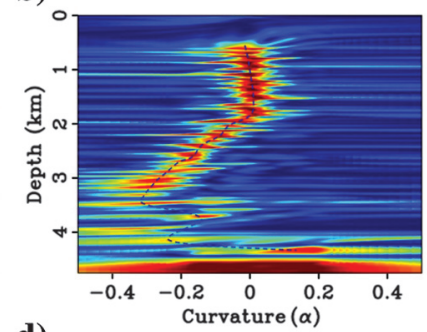

d)

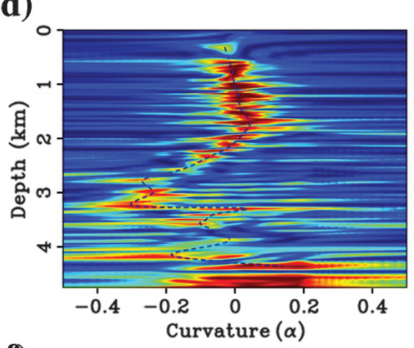

f)

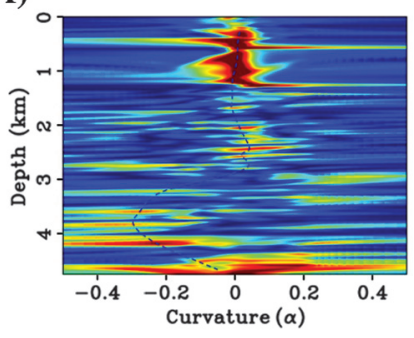

Figure 13. Semblance spectra calculated from Figure 12 after the first iteration.

$$
\begin{gathered}
\gamma_{2}\left(\mathbf{x}^{\prime}\right)=\frac{2}{c\left(\mathbf{x}^{\prime}\right)^{3}} \\
\times \sum_{\mathbf{x}_{s}} \sum_{\mathbf{x}} \int \overbrace{\left[\frac{\Delta \tau}{E} \ddot{p}_{g}\left(\mathbf{x}, t \mid x_{s}\right) * g\left(\mathbf{x}^{\prime},-t \mid \mathbf{x}, 0\right)\right]}^{\text {back propagation of the redatumed data }} \\
\overbrace{\left[\dot{p}_{s}\left(\mathbf{x}^{\prime}, t+\Delta \tau \mid \mathbf{x}_{s}\right)\right]}^{\text {forward propagation of the source }} d t .
\end{gathered}
$$

In the angle domain, the WT gradient is given by

$$
\gamma\left(\mathbf{x}^{\prime}\right)=\gamma_{1}\left(\mathbf{x}^{\prime}\right)+\gamma_{2}\left(\mathbf{x}^{\prime}\right)
$$

where

$$
\begin{aligned}
& \gamma_{1}\left(\mathbf{x}^{\prime}\right)=\frac{2}{c\left(\mathbf{x}^{\prime}\right)^{3}} \sum_{\boldsymbol{\theta}} \sum_{\mathbf{x}} \frac{\Delta \tau(\mathbf{x}, \theta)}{E} \int d \mathbf{x}_{s} \int d h \int \dot{p}_{g}\left(\mathbf{x}^{\prime}, t \mid \mathbf{x}_{s}\right) \\
& {\left[\ddot { p } _ { s } ( x - h , z + h \operatorname { t a n } \theta , t + \Delta \tau | \mathbf { x } _ { s } ) * g \left(\mathbf{x}^{\prime}, t \mid x+h, z\right.\right.} \\
& +h \tan \theta, 0)] d t
\end{aligned}
$$

and a)

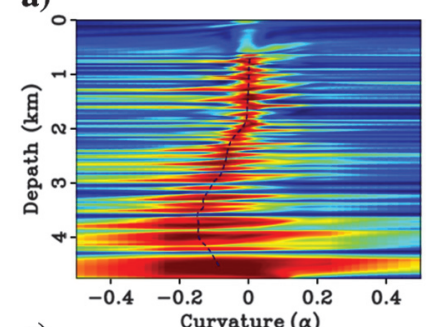

c)

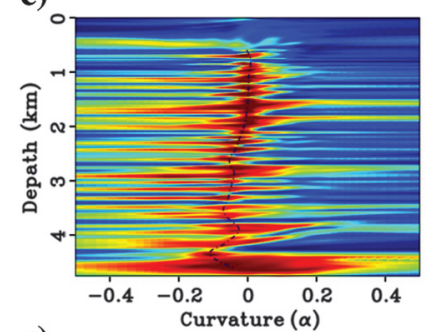

e)

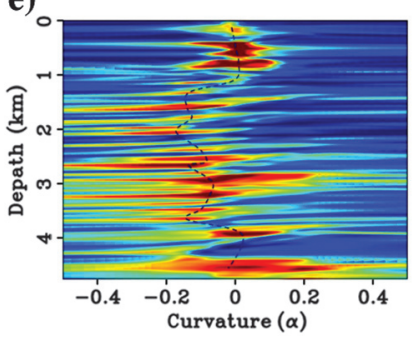

b)

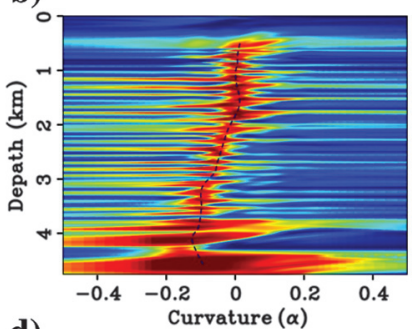

d)

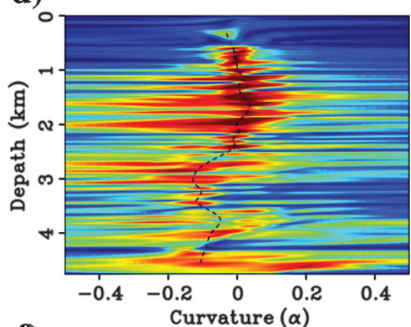

f)

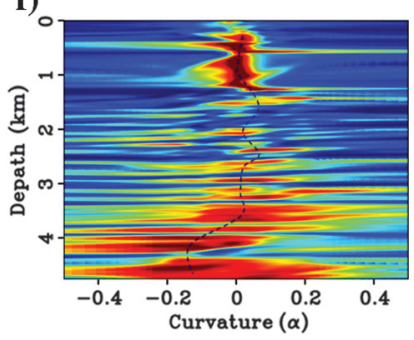

Figure 14. Semblance spectra calculated from the angle-domain crosscorrelation after 10 iterations. 


$$
\begin{aligned}
& \gamma_{2}\left(\mathbf{x}^{\prime}\right)=\frac{2}{c\left(\mathbf{x}^{\prime}\right)^{3}} \sum_{\theta} \sum_{\mathbf{x}} \frac{\Delta \tau(\mathbf{x}, \theta)}{E} \int d \mathbf{x}_{s} \\
& \times \int d h \int \dot{p}_{s}\left(\mathbf{x}^{\prime}, t+\Delta \tau \mid \mathbf{x}_{s}\right) \\
& {\left[\ddot{p}_{g}\left(x+h, z+h \tan \theta, t \mid \mathbf{x}_{s}\right) * g\left(\mathbf{x}^{\prime},-t \mid x-h, z+h \tan \theta, 0\right)\right] d t,}
\end{aligned}
$$

a)
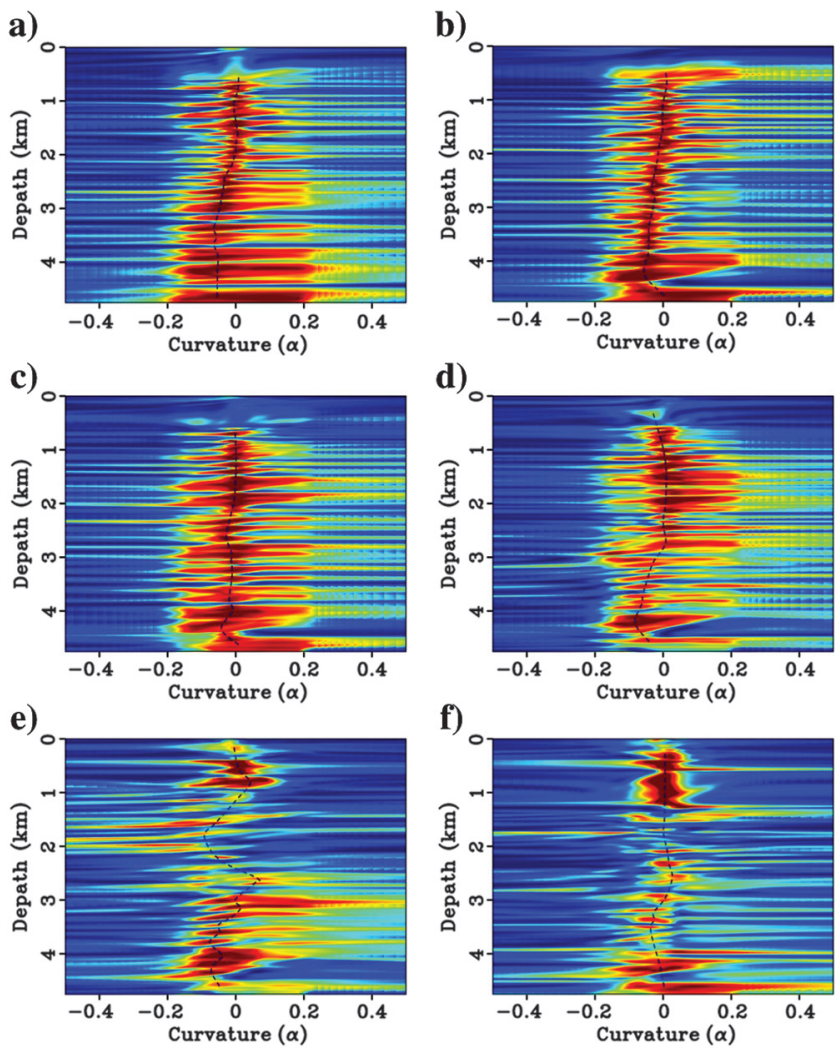

Figure 15. Semblance spectra calculated from the angle-domain crosscorrelation after 15 iterations.

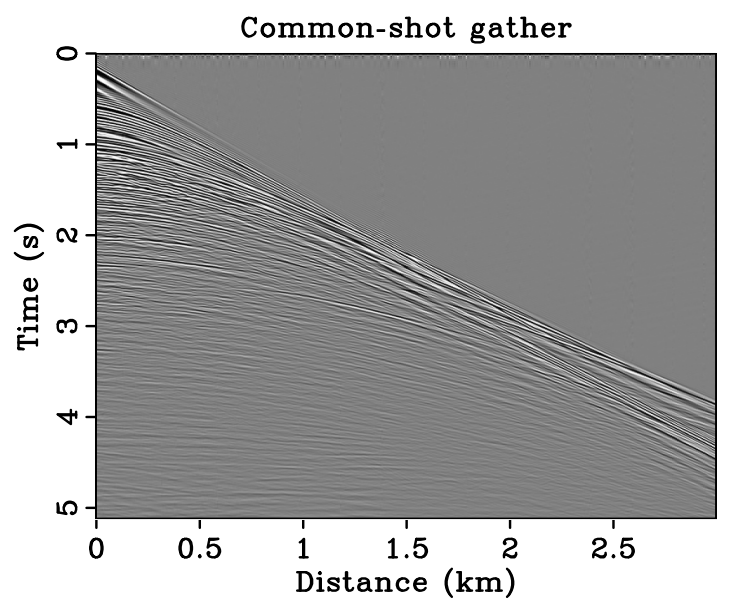

Figure 16. A common-shot gather from the Gulf of Mexico data. where $E$ is defined to be $\frac{\partial \dot{f}_{\Delta \tau}}{\partial \Delta \tau}$

$$
E=\frac{\partial \dot{f}_{\Delta \tau}}{\partial \Delta \tau} .
$$

The detailed derivation of these formulas is given in Zhang et al. (2015).

\section{NUMERAL EXAMPLES}

This section presents the results of applying WT to two synthetic data sets and one field data example. The first synthetic example is to test the effectiveness of shot-domain WT for a fault model. The second synthetic example illustrates that angle-domain WT is a robust method for updating the subsalt velocity model. Finally, a field data set is inverted by the angle-domain WT method to provide a more accurate velocity model than traveltime tomography below the depth of the diving wave. WT also provides a more accurate velocity update than FWI for this data set.

\section{Fault model}

The fault model in Figure 1a is a $250 \times 607$ grid of velocity values with 121 sources and 607 receivers evenly distributed on the top surface. An absorbing sponge zone with a width of 40 grid points is added along each side of the computational model and the grid interval is $10 \mathrm{~m}$. The bandwidth of the source wavelet is from 10 to $60 \mathrm{~Hz}$, and the observed data are generated by an eighth-order finite-difference solution to the $2 \mathrm{D}$ acoustic-wave equation with a constant density. Figure $1 \mathrm{~b}$ is a typical shot gather recorded on the free surface, where the direct wave is removed. The starting velocity model is shown in Figure 2a, which is a strongly smoothed version of the true model. Figure $3 \mathrm{a}$ and $3 \mathrm{~b}$ displays the shot-domain crosscorrelation functions computed with the starting velocity
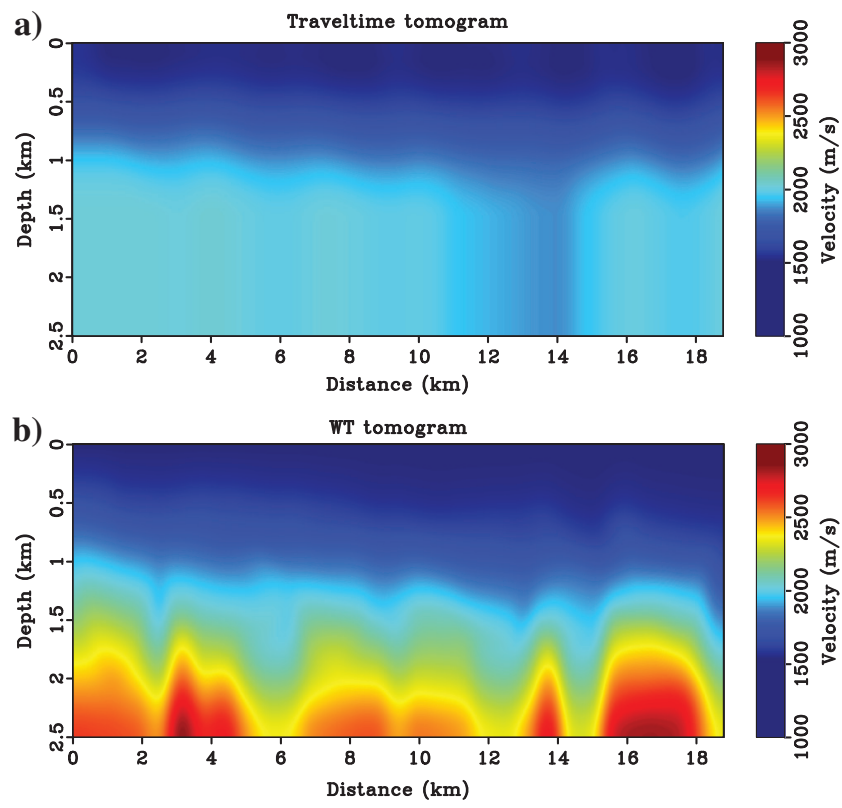

Figure 17. (a) The initial velocity model computed by first-arrival traveltime tomography and (b) the inverted velocity model after 13 iterations. 
model at the offsets 1.7, 2.7, 3.7, and $4.7 \mathrm{~km}$ in Figure 1a. The front surface of the 3D data cube in these figures shows the zero-lag crosscorrelation function, which is a display of the shot-domain CIGs. The unflattened curves in these CIGs indicate that the velocity model is not sufficiently accurate for migration. The depth of the reflectors is picked from the CIGs, and the shot-domain crosscorrelation function at this depth is obtained from the horizontal surface of the data cube. The top slice is the shot-domain crosscorrelation function. The side slice is the crosscorrelation function for a single shot. The reflection-traveltime residual curve is estimated by semblance scanning for the horizontal surface using equation 5 . Figure $4 \mathrm{a}$ and $4 \mathrm{~b}$ displays the semblance spectrum after the semblance scanning of the shot-domain crosscorrelation function in Figure $3 \mathrm{a}$ and $3 \mathrm{~d}$. In these figures, the deviation of the energy cluster from zero curvature indicates a reflection-traveltime curve residual at the indicated depth. The analytical calculation of the reflectiontraveltime residual using equation 5 can be obtained so long as the curvature is picked from the semblance spectrum in Figure $4 \mathrm{a}$ and $4 \mathrm{~d}$. After the velocity update at each iteration, the shot-domain crosscorrelation functions and the semblance spectrum are recalculated, and the new reflection-traveltime residual is estimated for the new velocity update at the next iteration.

Inversion results after 6 and 10 iterations are displayed in Figure $2 \mathrm{~b}$ and $2 \mathrm{c}$. It is clear that details in the true model, such as the fault and the thin layer, become visible in Figure $2 c$. The corresponding semblance spectra are shown in Figures 5a and 6d. From these figures, we can see that after the tenth iteration, the energy cluster in the semblance spectrum focuses at zero curvature as shown in Figure $6 a$ and $6 \mathrm{~d}$. This means the reflection events in the CIGs become flatter and the most significant energy is focused at zero time lag. The RTM images based on the initial velocity model and the inverted velocity model are displayed in Figure $7 \mathrm{a}$ and $7 \mathrm{c}$. Figure 7c is the RTM image after the tenth iteration, which accurately images the reflectors and the fault.

Figure 8a displays the FWI tomogram after 200 iterations with the same starting velocity model. Low frequencies are missing in a)

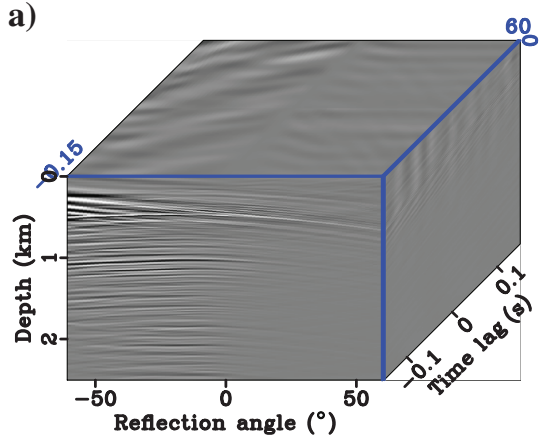

d)

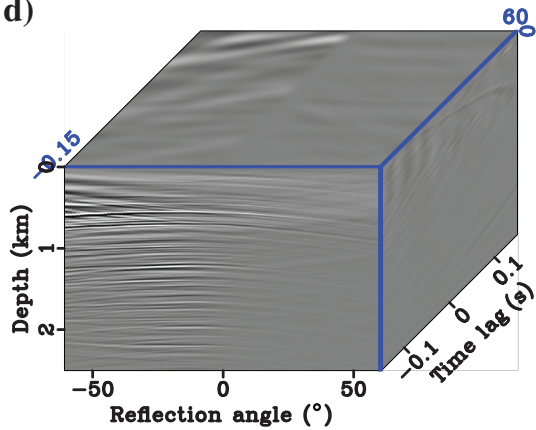

g)

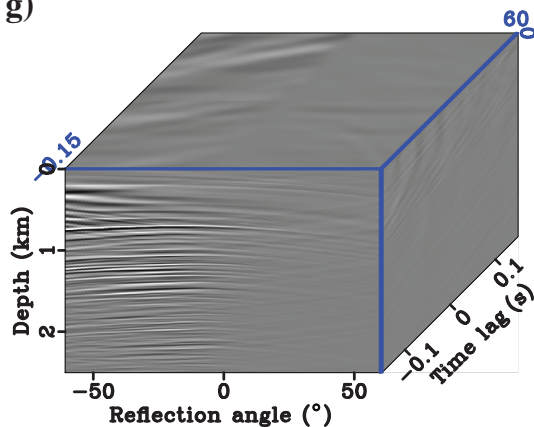

b)

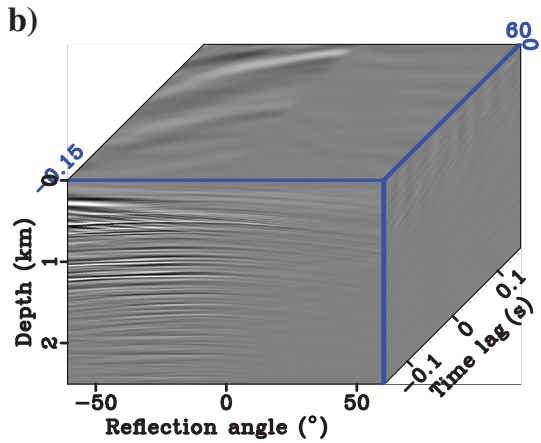

e)

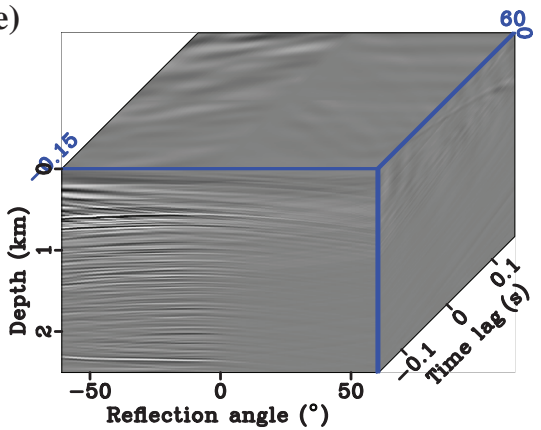

h)

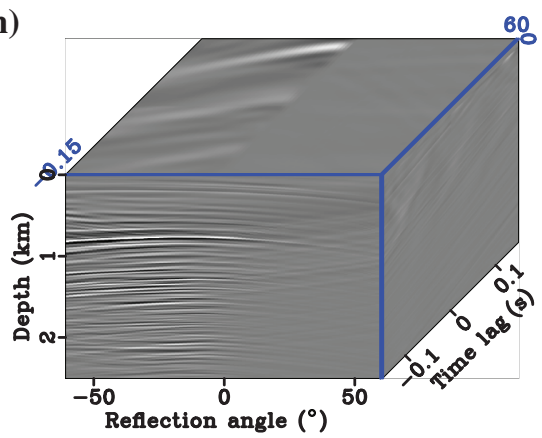

c)

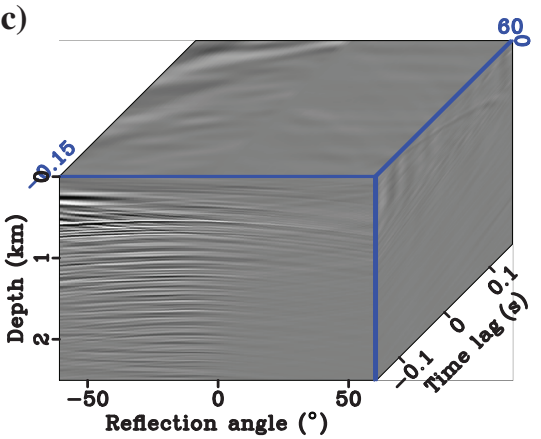

f)

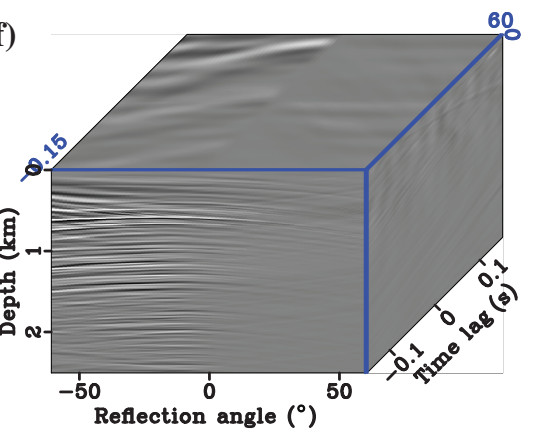

i)

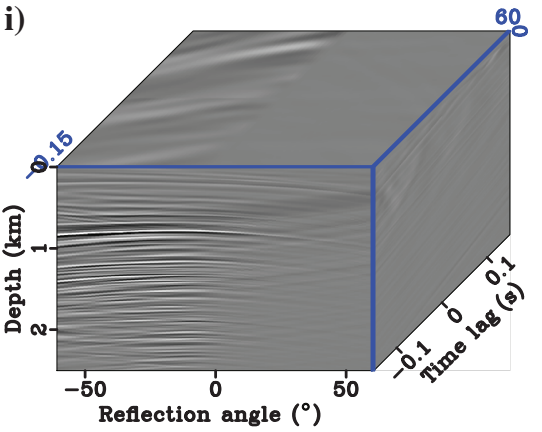

Figure 18. Angle-domain crosscorrelation functions generated from the initial velocity model using RTM. The horizontal locations of each figure are from 2 to $18 \mathrm{~km}$ in the velocity model. 
the observed data, so FWI fails to accurately image the model, especially in the deeper sections. Figure $8 \mathrm{~b}$ shows the associated RTM image with the FWI tomogram. Compared with the FWI tomograms, the WT tomogram provides a more accurate velocity to migrate the data from the shallow to the deep zones. This is because FWI could not provide a robust velocity update due to cycle skipping, whereas WT mitigates the cycle-skipping problem (Zhang et al., 2015).

\section{Sigsbee salt model}

The second test inverts synthetic data computed for the Sigsbee salt model. The true model shown in Figure 9a is discretized into a mesh with $476 \times 1000$ grid points, and the grid point interval is $10 \mathrm{~m}$. The source is a Ricker wavelet with a peak frequency of $20 \mathrm{~Hz}$. The observed seismograms are generated by an eighth-order finite-difference solution to the $2 \mathrm{D}$ acoustic-wave equation with constant density. Figure $9 \mathrm{~b}$ displays a shot gather recorded on the free surface. The region surrounded by the rectangle in the starting velocity model shown in Figure 10a is $80 \%$ of the true velocity model, where RTM is used to migrate the observed data with this starting velocity model. The migration image in Figure 11a indicates that the reflectors beneath the salt are not properly imaged due to an inaccurate velocity.

The angle-domain crosscorrelation functions calculated from the starting velocity model in Figure 9a are displayed in Figure 12a and 12f. The locations of the angle-domain crosscorrelation computed with equation 2 are indicated by the six lines in Figure 9a. The front surface of these data cubes displays the angle-domain CIGs, and the horizontal surface depicts the angle-domain crosscorrelation functions. The side slice is the crosscorrelation function for a single angle. Figure $13 \mathrm{a}$ and $13 \mathrm{f}$ is the semblance images scanned for the best-fitting curvatures in the angle-domain crosscorrelation functions computed with equation 6 . The clustering of maximum amplitude values indicates a time-shift curve for the optimal angle-domain crosscorrelation function. The deviation of these en- a)

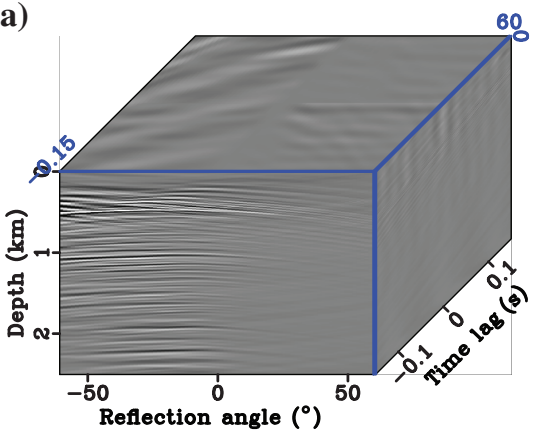

d)

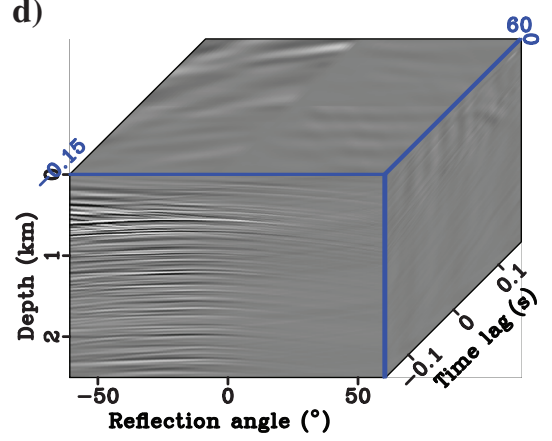

g)

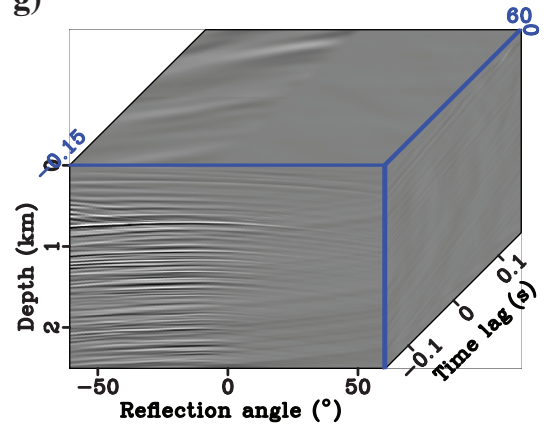

b)

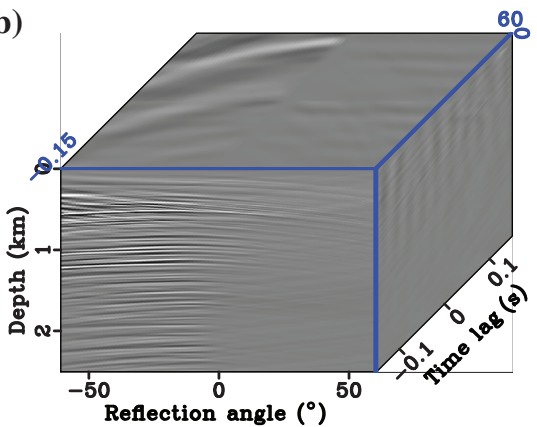

e)

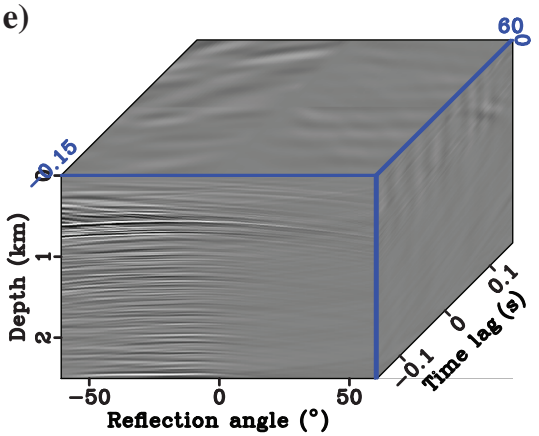

h)

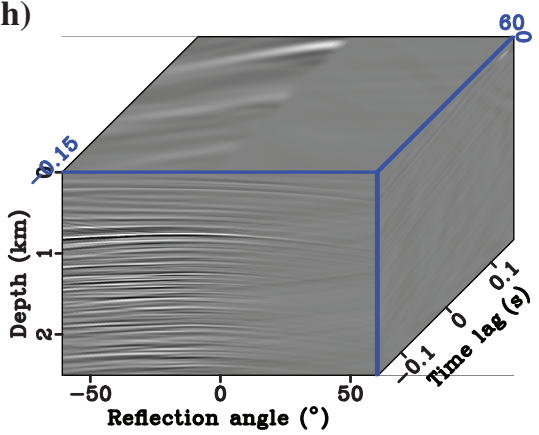

c)

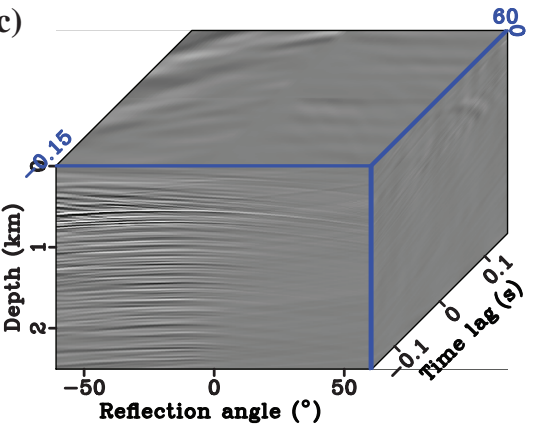

f)

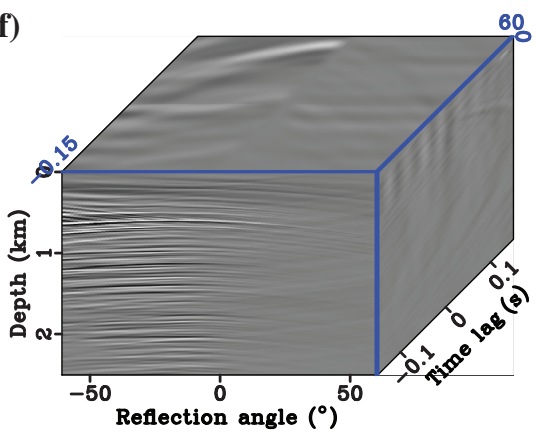

i)

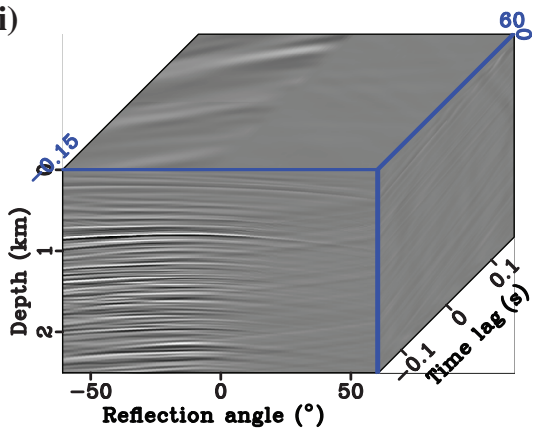

Figure 19. Angle-domain crosscorrelation functions generated from the inverted velocity model using RTM. The horizontal locations of each figure are from 2 to $18 \mathrm{~km}$ in the velocity model. 
ergy clusters from zero indicates errors in the velocity model. Figure $10 \mathrm{~b}$ and $10 \mathrm{c}$ shows the inversion results after 10 and 15 iterations, and the corresponding semblance spectra are shown in Figures $14 \mathrm{a}$ and $15 \mathrm{f}$. The clustering of maximum energy values around zero curvature implies that the time shift is close to zero, and the inverted velocity becomes more accurate. Another validity check is the RTM images shown in Figure 11a and 11c. They illustrate that the final inverted velocity model can be used to accurately image the reflectors beneath the salt.

\section{Gulf of Mexico data}

The third example is for marine data recorded in the Gulf of Mexico. These streamer data are acquired using 496 shots with a shot interval of $37.5 \mathrm{~m}$, a time-sampling interval of $1 \mathrm{~ms}$, a trace length of $5 \mathrm{~s}$, and 480 active hydrophones per shot. The grid interval is $6.25 \mathrm{~m}$, and the hydrophone intervals are between 11 and $13 \mathrm{~m}$, with a near source-receiver offset of $198 \mathrm{~m}$ and a far offset of
$6.184 \mathrm{~km}$. The source wavelet is estimated by stacking along the first arrival at the nearest offset of each shot, and the data are low-pass-filtered between 5 and $45 \mathrm{~Hz}$. Figure 16 shows a common-shot gather after filtering. The initial velocity model is obtained using first-arrival traveltime tomography, and the tomogram is shown in Figure 17a. The angle-domain crosscorrelation functions are then calculated from the initial velocity model and are displayed in Figure 18a and 18i. From these figures, we can see that the first-arrival traveltime tomogram provides an accurate velocity model that mostly flattens the CIGs no deeper than $2 \mathrm{~km}$. This is because the turning waves are unable to penetrate deeper than $2 \mathrm{~km}$ for the $6 \mathrm{~km}$ source-receiver offset. We now apply WT to the deep reflection events to improve the velocity in the deeper parts of the initial velocity model.

The reflection-traveltime residuals are estimated by picking the maximum energy in the semblance scans of the angle-domain crosscorrelation functions. The inverted velocity model is displayed in Figure 17b. Figure 19a and 19i shows the angle-domain crosscor-

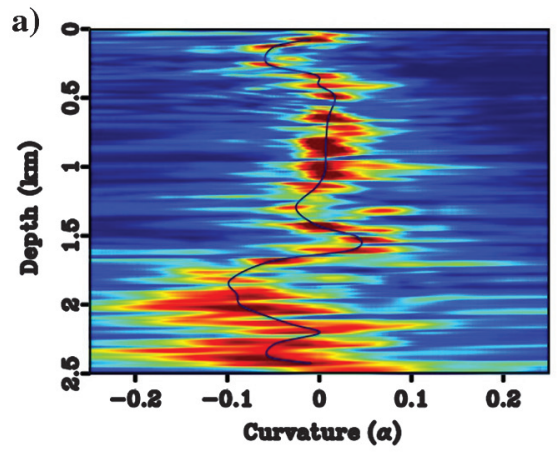

d)
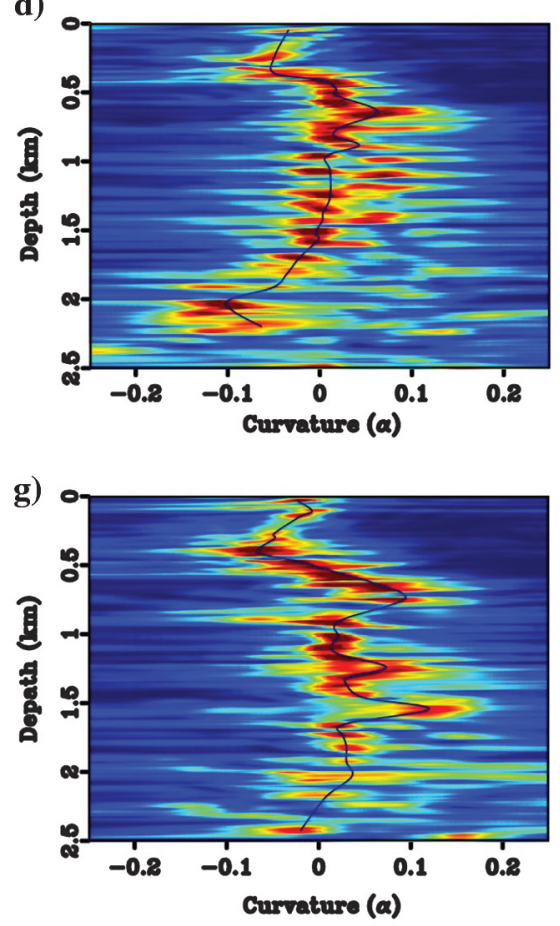

b)

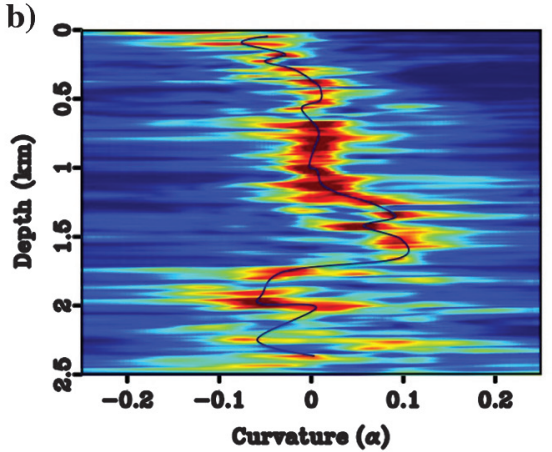

e)

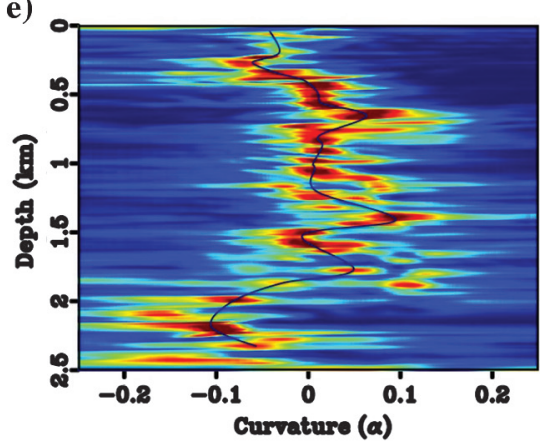

h)

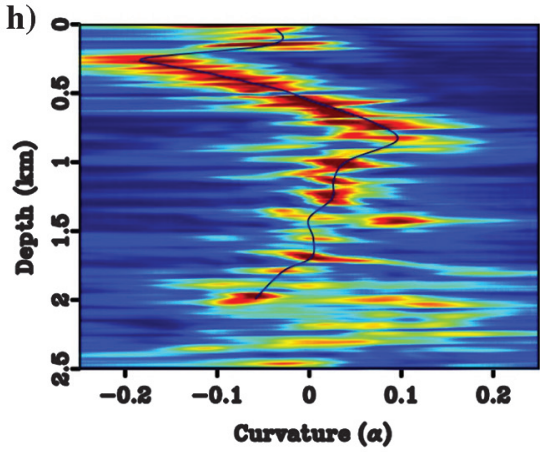

c)

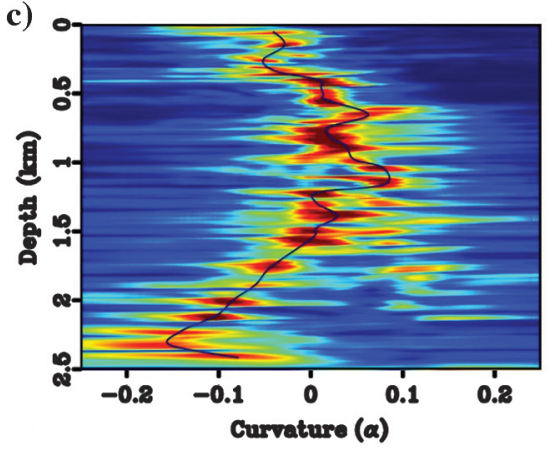

f)

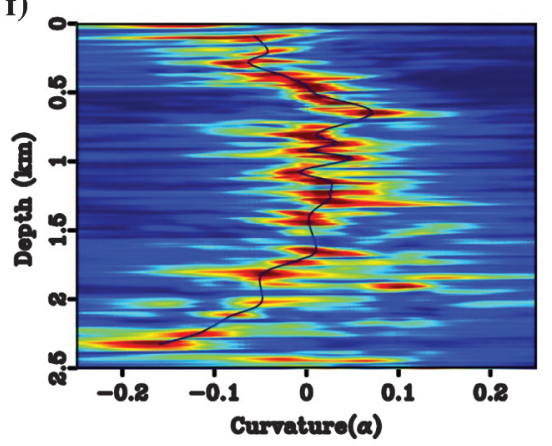

i)

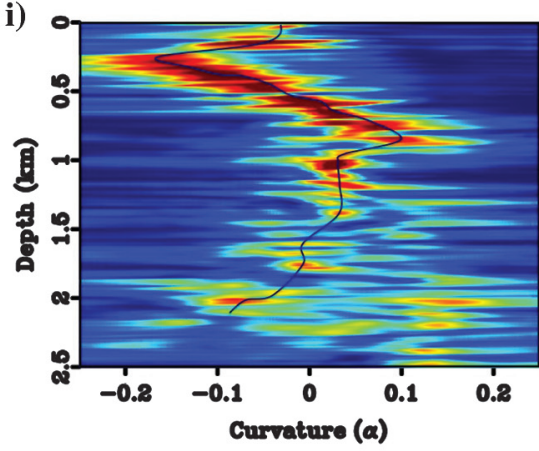

Figure 20. Semblance spectra calculated from Figure 18. 
relation functions calculated from the inverted velocity model, in which the angle-domain CIGs are flattened at depths greater than $2 \mathrm{~km}$ compared with the unflattened CIGs in Figure 18a and 18i. Most of the maximum energy in the semblance scanning focuses at zero curvature as shown in Figure 21a and 21i compared with that shown in Figure 20a and 20i, which means that the reflectiontraveltime residuals are nearly zero.

We now compare the RTM images from the different velocity models. The RTM images using the velocity models from traveltime tomography and angle-domain WT are displayed in Figure 22a and 22b. The magnified views of the migration images are shown in Figure $23 \mathrm{a}$ and $23 \mathrm{f}$. It is obvious that the WT images are better focused than those obtained by traveltime tomography.

Figure 24a displays the FWI tomogram computed from the same data set and the starting velocity model (Boonyasiriwat et al., 2009). The associated RTM image is shown in Figure 24b. It is clear that the WT tomogram provides a more accurate velocity model for imaging deep reflections. Figures $25 \mathrm{a}$ and $26 \mathrm{c}$ show the angle-domain CIGs calculated from the traveltime, FWI, and WT tomograms. It is obvious that the WT tomogram flattens the angle-domain CIGs in the deeper part of the model, whereas the traveltime and FWI tomograms fail to flatten the angle-domain CIGs. However, there are portions in the deeper parts of the model in which the CIGs are not flattened. This highlights the fact that the time lags can be misestimated if the reflection events are weak or if they are surrounded by many other strong events.

\section{DISCUSSION}

Compared with shot-domain WT, angle-domain WT has benefits and limitations in its practical applications. The first limitation is that angle-domain WT is much more computationally expensive than shot-domain WT. This is because we need to compute the subsurface offset-domain crosscorrelation functions over space and time lags. The second limitation is that when the acquisition geometry might not be as favorable to the creation of high-quality angle gathers, in particular for 3D, angle-domain WT does not always work better than shot-domain WT. One benefit is that angle-domain a)
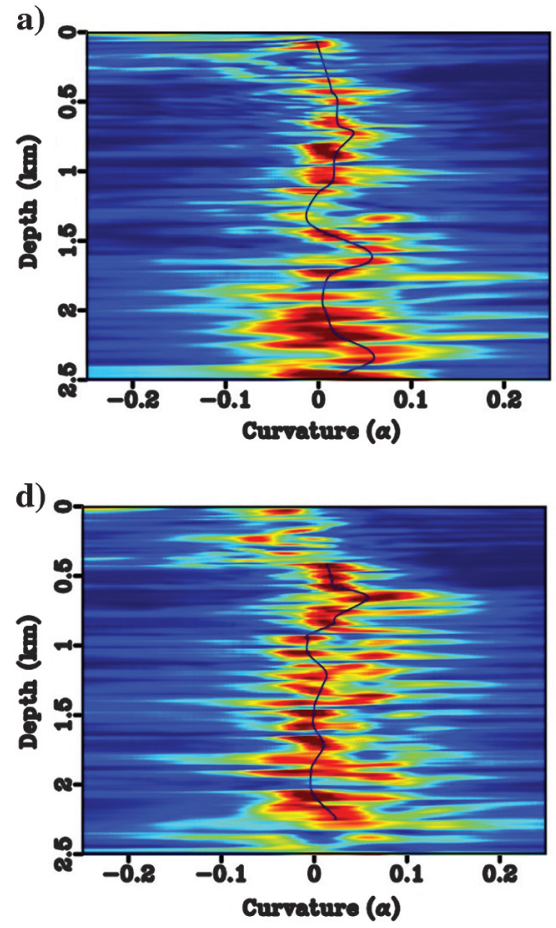

g)

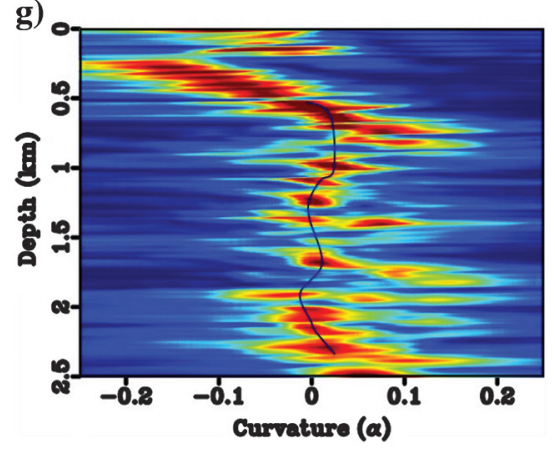

b)

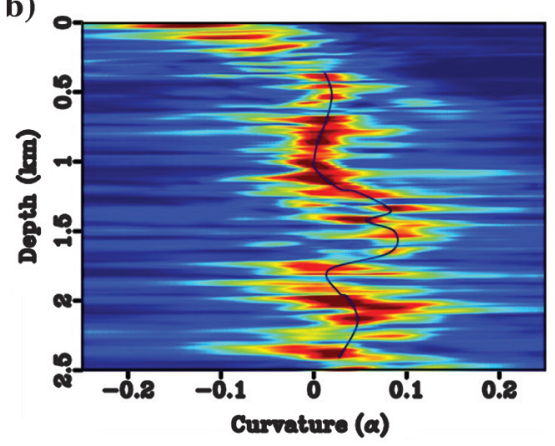

e)

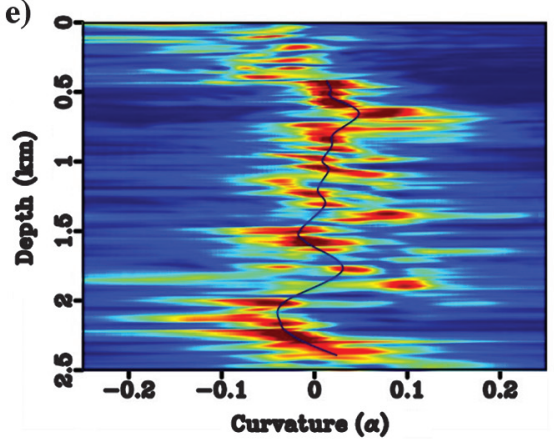

h)

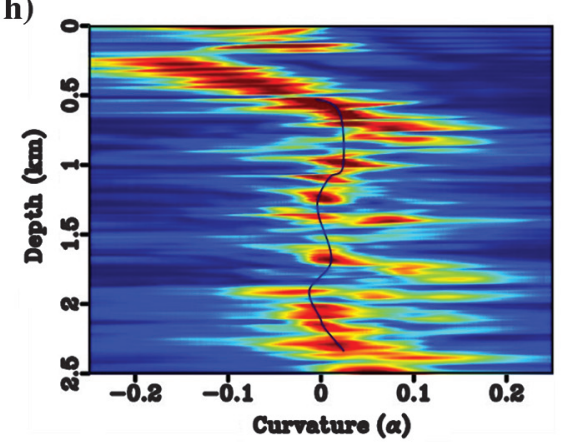

c)

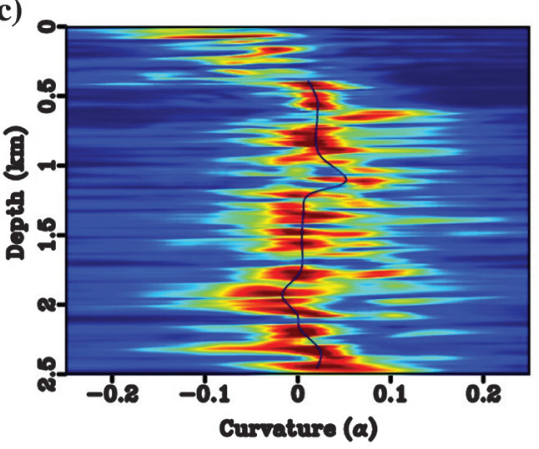

f)

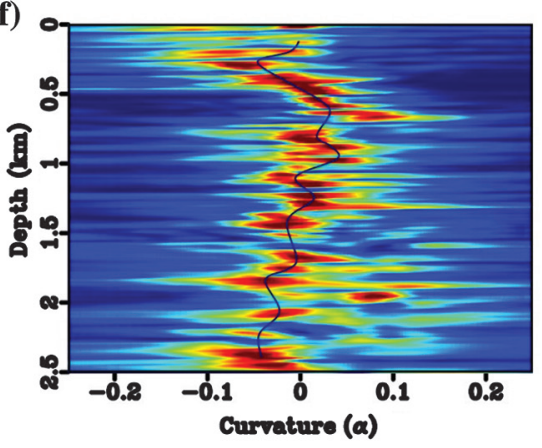

i)

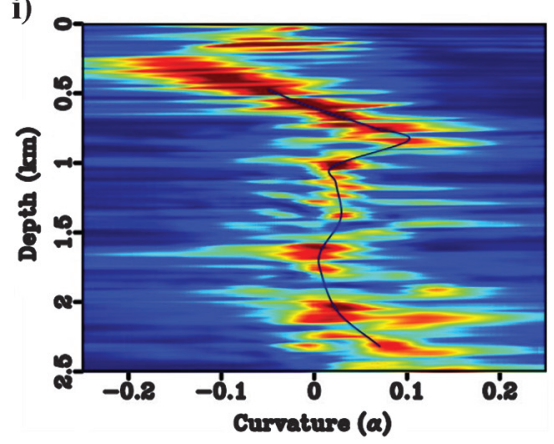

Figure 21. Semblance spectra calculated from Figure 19. 

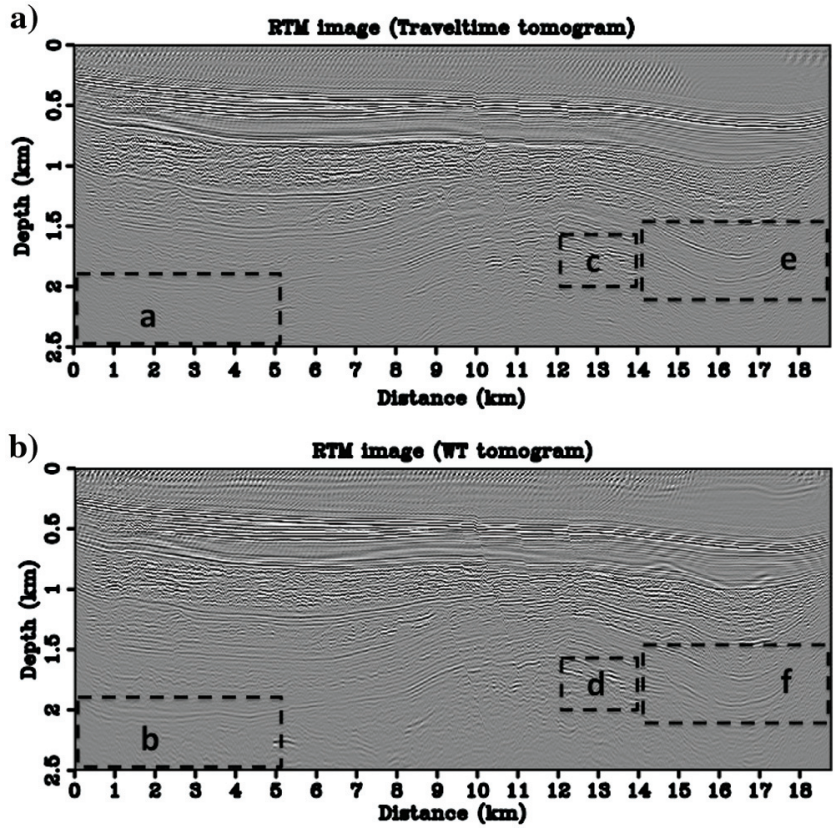

Figure 22. ( $a$ and b) RTM images using the initial velocity model and the WT tomogram in Figure 17, respectively. The rectangles show the magnified areas in Figure 23.
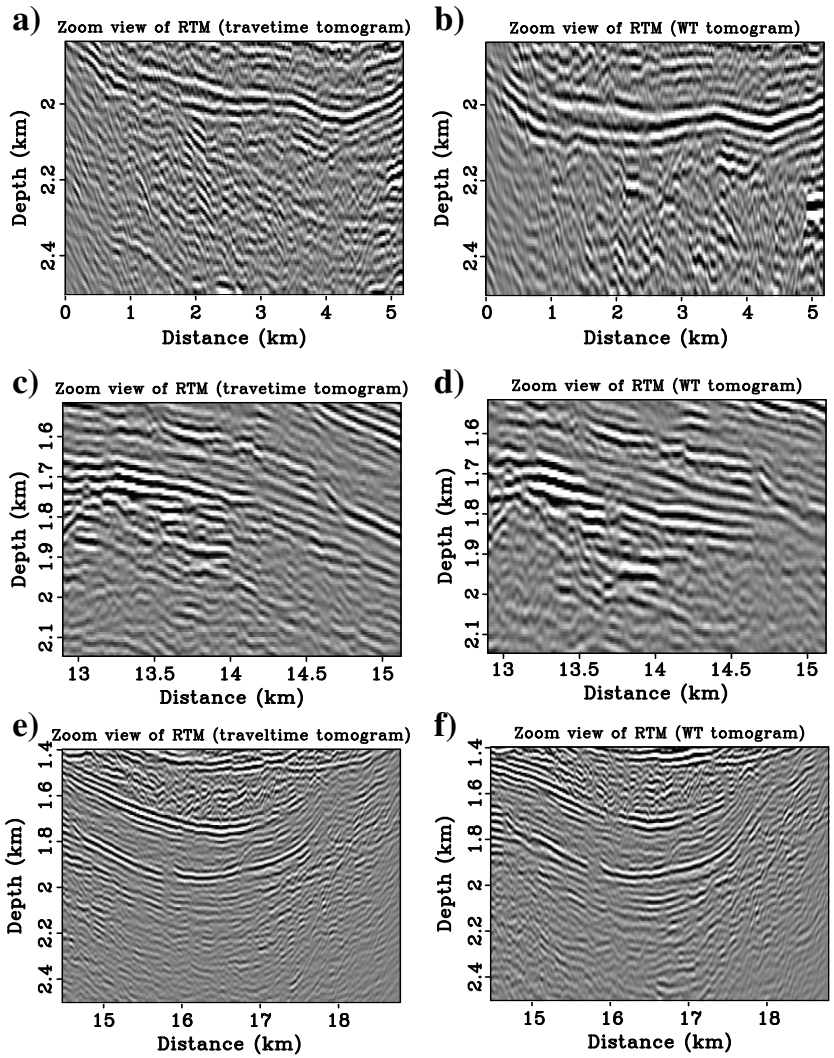

Figure 23. (a, c, and e) Magnified views from Figure 22a and (b, d, and f) magnified views from Figure $22 \mathrm{~b}$.
WT is more robust for complex models than shot-domain WT. The main reason is that the angle-domain crosscorrelation function can estimate the traveltime residual more robustly than the shot-domain crosscorrelation function. Angle-domain crosscorrelation has fewer artifacts and is more coherent than shot-domain crosscorrelation for complex structures. This is the main motivation for using the angledomain WT method for inversion of the subsalt velocity model, whereas shot-domain WT is only applied to the layered model in this paper. It is easier to estimate the traveltime residual with angle-domain crosscorrelation than shot-domain crosscorrelation. Another benefit is that the multiarrivals can be accounted for with angle-domain WT. In contrast, shot-domain WT only takes into account single arrivals.

For the 2D examples shown in this paper, the computation can be affordable in practice. For the $3 \mathrm{D}$ case, the computation cost is mainly for the shot- and angle-domain crosscorrelations. The computational cost of the WT method largely depends on the number of the image points and the time or space lags chosen for the inversion. More image points are required to improve the resolution of the inverted velocity model. Generally speaking, shot- and angle-domain WT are much more expensive than FWI. This is because the WT method needs to calculate the shot- and angle-domain crosscorrelations, which can be computationally expensive. In practice, you do not need to calculate the shot- or angle-domain crosscorrelation for the whole space. Only a limited number of points are needed for the crosscorrelation and inversion.

One limitation of the WT method is that it is not an automatic method, and manual picking is sometimes required to ensure a robust estimate of traveltime residuals. For complex models with short-wavelength errors, the semblance analysis based on a single parameter moveout equation does not ensure a precise representation of traveltime residuals. In this case, multiparameter moveout semblance analysis, predictive painting (Fomel, 2010), and time warping (Burnett and Fomel, 2009) are possible approaches for
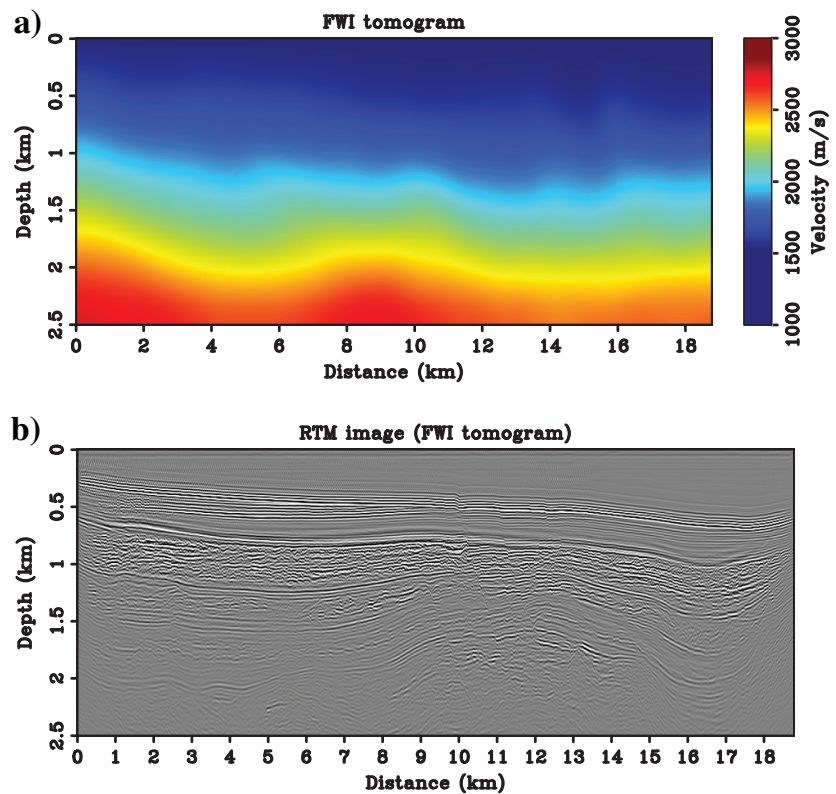

Figure 24. ( $a$ and b) Depiction of the FWI tomogram and the associated RTM image. 

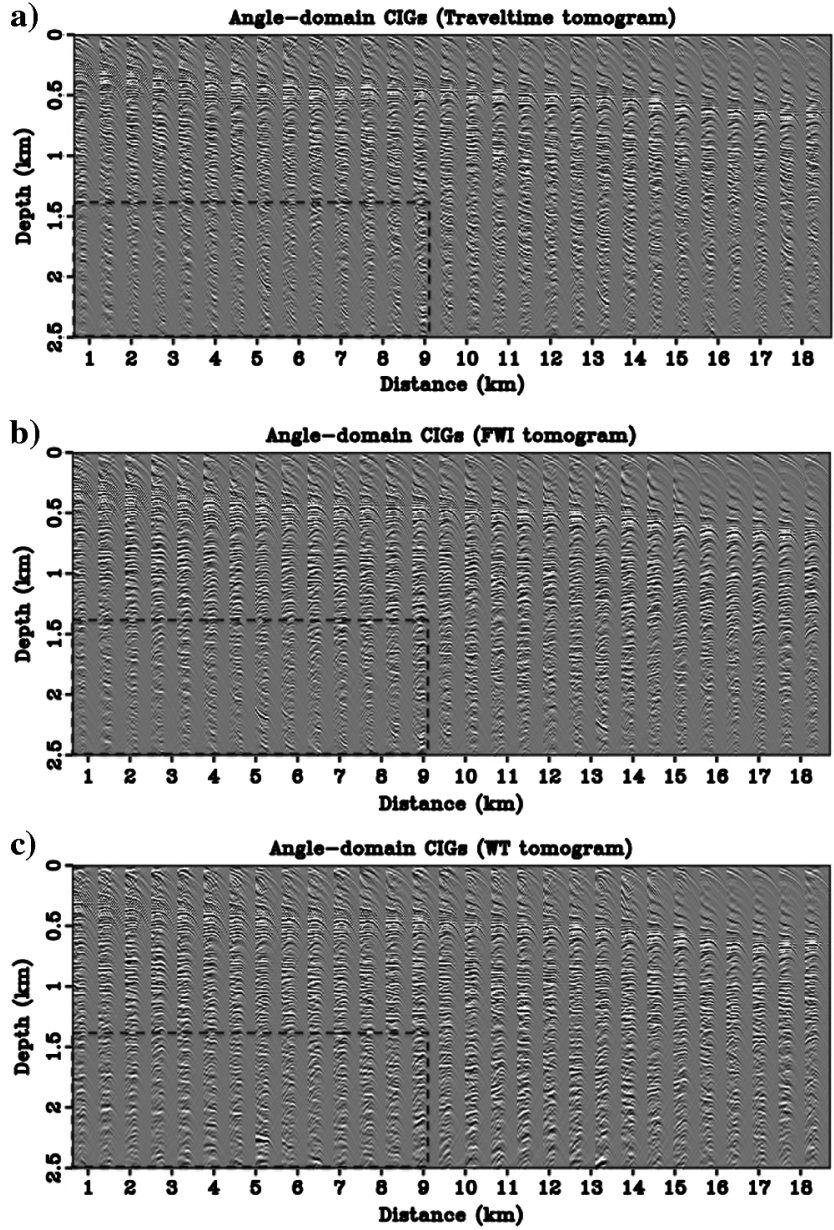

Figure 25. The angle-domain CIGs are calculated from (a) the firstarrival traveltime tomogram, (b) the FWI tomogram, and (c) the WT tomogram, respectively. Three rectangles indicate the magnified view area shown in Figure 26.

extracting the traveltime residuals. This is a subject for future research.

\section{SUMMARY}

Three numerical examples are shown to verify the effectiveness and limitations of shot- and angle-domain WT. The key to success for WT is to accurately estimate the reflection-traveltime residual. Results show that the angle-domain crosscorrelation function is more robust than the shot-domain crosscorrelation function for estimating the traveltime residual in complex models. Even for complex velocity models, such as some subsalt models, the angledomain crosscorrelation function can sometimes provide a robust estimate of the reflection-traveltime residual for updating the velocity model. However, the computation of the angle-domain crosscorrelation function is much more expensive than the shot-domain crosscorrelation function. Synthetic data tests illustrate that WT is effective for inverting the velocity model from reflection data associated with complex velocity models. Results with Gulf of Mexico data demonstrate that WT can be used to update the deeper portions of the velocity model, which are not accessible to turning waves. A limitation of the WT method is that it can require signifi-
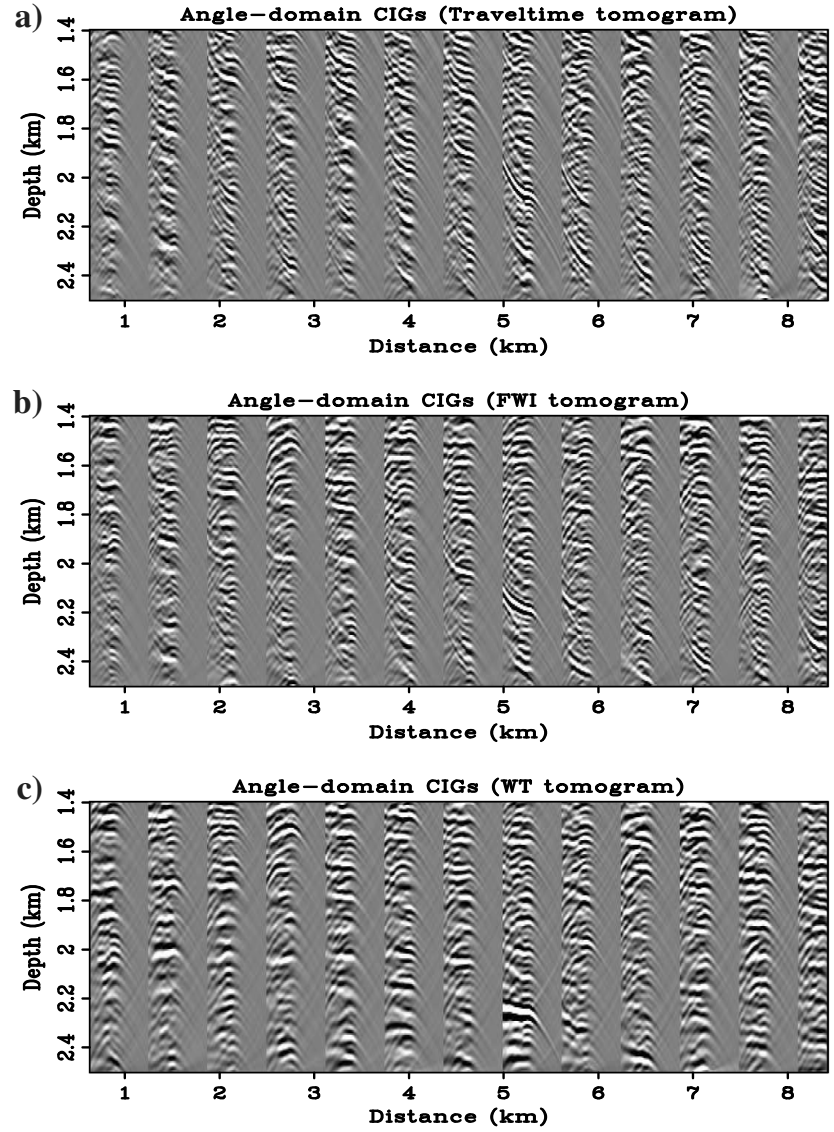

Figure 26. Magnified view of angle-domain CIGs calculated from the (a) first-arrival traveltime tomogram, (b) FWI tomogram, and (c) WT tomogram, respectively. The locations are indicated by the rectangles in Figure 25.

cant user intervention, and it is computationally intensive for $3 \mathrm{D}$ data. Another problem is that the correlation can be misestimated if the reflection events are weak or if they are surrounded by many other strong events.

\section{ACKNOWLEDGMENTS}

We acknowledge the sponsors of the Center for Subsurface Imaging and Fluid Modeling at KAUST for their support. We also appreciate the KAUST Supercomputing Lab for the use of the Shaheen supercomputer and the information technology department for their hardware support. Special acknowledgments go to IBM for the use of their Blue Gene/Q computer in New York. We are thankful for the support of a KAUST CRG grant. We appreciate the suggestions and comments from J. Etgen, J. Shragge, and some other reviewers.

\section{REFERENCES}

Almomin, A., 2011, Correlation-based wave-equation migration velocity analysis: 81st Annual International Meeting, SEG, Expanded Abstracts, 3887-3891.

Al-Yahya, K., 1987, Velocity analysis by iterative profile migration: Ph.D. thesis, Stanford University.

Boonyasiriwat, C., P. Valasek, P. Routh, W. Cao, G. Schuster, and B. Macy, 2009, An efficient multiscale method for time-domain waveform tomography: Geophysics, 74, no. 6, WCC59-WCC68, doi: 10.1190/1.3151869. 
Burnett, W. and S. Fomel, 2009, Moveout analysis by time-warping: 79th Annual International Meeting, SEG, Expanded Abstracts, 3710-3714.

Fomel, S., 2010, Predictive painting of 3-D seismic volumes: Geophysics, 75, no. 4, A25-A30, doi: 10.1190/1.3453847.

Luo, Y., and G. Schuster, 1991, Wave equation traveltime inversion: Geophysics, 56, 645-653, doi: 10.1190/1.1443081.

Sava, P., and B. Biondi, 2004, Wave-equation migration velocity analysis. Part I: Theory: Geophysical Prospecting, 52, 593-606, doi: 10.1111/j .1365-2478.2004.00447.x.

Shen, P., and W. Symes, 2008, Automatic velocity analysis via shot profile migration: Geophysics, 73, no. 5, VE49-VE59, doi: 10.1190/1.2972021.

Symes, W., and M. Kern, 1994, Inversion of reflection seismograms by differential semblance analysis: Algorithm structure and synthetic examples: Geophysical Prospecting, 42, 565-614, doi: 10.1111/j.1365-2478.1994 .tb00231.x.

Wang, B., C. Mason, M. Guo, K. Yoon, J. Cai, J. Ji, and Z. Li, 2009, Subsalt velocity update and composite imaging using reverse-time-migration based delayed-imaging-time scan: Geophysics, 74, no. 6, WCA159WCA166, doi: 10.1190/1.3227152.
Yang, T., and P. Sava, 2011, Wave-equation migration velocity analysis with time-lag imaging: Geophysical Prospecting, 59, 635-650, doi: 10.1111/J 1365-2478.2011.00954.x.

Yang, T., and P. Sava, 2013, 3D image-domain wavefield tomography using time-lag extended images: 83rd Annual International Meeting, SEG, Expanded Abstracts, 4816-4821.

Zhang, S., Y. Luo, and G. Schuster, 2015, Shot- and angle-domain waveequation traveltime inversion of reflection data: Theory: Geophysics, 80, this issue, doi: 10.1190/geo2014-0178.1.

Zhang, S., and G. Schuster, 2012, Generalized differential semblance optimization: 75th Annual International Conference and Exhibition, Extended Abstracts, doi: 10.3997/2214-4609.20130374.

Zhang, S., G. Schuster, and Y. Luo, 2011, Wave-equation reflection traveltime inversion: 81st Annual International Meeting, SEG, Expanded Abstracts, 2705-2710, doi: 10.1190/1.3627756.

Zhang, S., G. Schuster, and Y. Luo, 2012, Angle-domain migration velocity analysis using wave-equation reflection traveltime inversion: 82nd Annual International Meeting, SEG, Expanded Abstracts, doi: 10.1190/ segam2012-1123.1. 